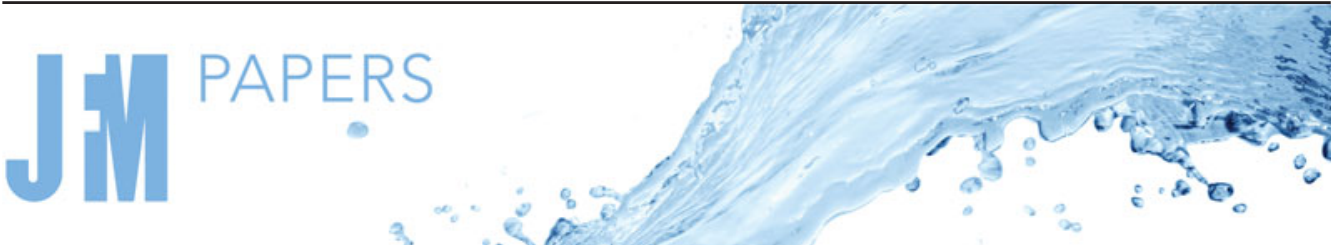

\section{On rotational flows with discontinuous vorticity beneath steady water waves near stagnation}

\author{
Lin Chen ${ }^{1,2}$, Biswajit Basu ${ }^{2} \uparrow$ and Calin-I. Martin ${ }^{3}$ \\ ${ }^{1}$ Department of Bridge Engineering, Tongji University, Shanghai 200092, PR China \\ ${ }^{2}$ School of Engineering, Trinity College Dublin, Dublin 2, Ireland \\ ${ }^{3}$ Department of Mathematics, University of Vienna, Vienna 1010, Austria
}

(Received 2 February 2020; revised 14 August 2020; accepted 16 November 2020)

\begin{abstract}
We numerically investigate the flow structure of periodic steady water waves of fixed relative mass flux propagating on rotational flows with piece-wise constant vorticity. We show that, for wave solutions along the global bifurcation diagram, the stagnation point can first occur internally or at the bottom, and then again occurs at the crest with further increase in wave height. We observe that the bifurcation diagram has a new branch which is not connected to the trivial solution. Furthermore, we present an in-depth discussion of the results on pressure distributions and particle trajectories beneath large-amplitude steady waves near stagnation. We also expand on previous results concerning the amplitude and mass flux of steady water waves travelling on rotational flows with discontinuous vorticity.
\end{abstract}

Key words: stratified flows, surface gravity waves, internal waves

\section{Introduction}

The interaction between surface water waves and underlying currents affects the wave properties significantly (Longuet-Higgins \& Stewart 1961), as also confirmed by experiments, e.g. Swan, Cummins \& James (2001). The interaction is hence of practical interest for design of offshore structures; see recent comparisons of structural responses with and without this interaction considered (Chen \& Basu 2018a,b) where linear wave-current interaction models were used, however; for coastal engineering applications (Kirby \& Dalrymple 1983; Dong \& Kirby 2012) and in geophysical fluid dynamics (Kirby 1984; Kirby \& Chen 1989). Of most interest is the problem of two-dimensional (2-D) periodic water waves propagating on a steady current. A large number of theoretical studies and numerical investigations have been dedicated to this problem. However, it is still far from being fully understood. A comprehensive introduction to nonlinear wave-current interactions can be found in Constantin (2011).

$\dagger$ Email address for correspondence: basub@tcd.ie

(C) The Author(s), 2021. Published by Cambridge University Press. This is an Open Access article, distributed under the terms of the Creative Commons Attribution licence (http://creativecommons.org/ licenses/by/4.0/), which permits unrestricted re-use, distribution, and reproduction in any medium, provided the original work is properly cited. 


\section{Chen, B. Basu and C.-I. Martin}

In fact, it is only recently that the existence of the large-amplitude wave solutions for rotational flows with general vorticity (thus allowing arbitrary current profiles) in finite water depth has been proved by Constantin \& Strauss (2004). Prior to this, only waves of small amplitude were known to exist in the presence of vorticity (Dubreil-Jacotin 1934). Hur (2006) further extended the proof of Constantin \& Strauss (2004) to the case of infinite depth with the additional restriction that the vorticity distribution is positive and monotone. Hur (2011) later completed the proof of existence of large-amplitude waves with infinite depth removing the mentioned restrictions on vorticity. Moreover, Constantin \& Strauss (2011) and Strauss (2012) addressed the existence of periodic travelling waves of small and large amplitude in a flow with an arbitrary bounded but discontinuous vorticity.

Following the work by Constantin \& Strauss (2004), rotational flows with constant and non-constant vorticity have received considerable attention. Notably, many numerical investigations, on both irrotational and rotational flows, appeared much earlier. For example, for irrotational flow in finite water depth, Chappelear (1961) expanded the velocity field and the wave profile in Fourier series and the Fourier coefficients were numerically determined from the Bernoulli equation by using the least squares method. The direct numerical simulation results were compared with the fifth-order Stokes theory. Dalrymple (1974a) presented a numerical model for steady water waves propagating over a linear-shear current (constant vorticity) where the streamfunction was expressed as the superposition of a linearly varying current profile and an irrotational wave field. The results thereof showed that the vorticity has a significant impact on the wave height. Simmen \& Saffman (1985) and Teles da Silva \& Peregrine (1988) studied periodic steady water waves with constant vorticity for infinite and finite water depth, respectively. The numerical methods for irrotational flows and rotational flows with constant vorticity are generally based on the boundary integral method or the Fourier expansion. Recently, Clamond \& Dutykh (2018) developed a novel conformal mapping method for irrotational flows, where the governing equation is rendered of Babenko kind which is then solved using the Petviashvili method. The model is very efficient and is able to solve for large-amplitude waves with quite large steepnesses over arbitrary depth. Dyachenko \& Hur (2019b) further modified the Babenko equation to permit constant vorticity and applied Newton-GMRES (generalized minimal residual) method following the use of the Fourier expansion. There are also other methods that have been developed. For instance, Ablowitz, Fokas \& Musslimani (2006) proposed a non-local formulation which was further applied to consider non-flat bottom and moving non-flat bottom boundaries (Fokas \& Nachbin 2012; Francius \& Kharif 2017). Wahlén (2009) and Ehrnström, Escher \& Wahlén (2011) devised a novel transform for small-amplitude waves with constant and linear vorticity, i.e. a vertical scaling transformation.

For waves on rotational flows with non-constant vorticity, early numerical investigations date back to Dalrymple $(1973,1977)$, where the author considered the effects of various current profiles on the finite-amplitude steady waves over finite water depth. The method was based on the Dubreil-Jacotin (DJ) transformation and finite difference discretization of the transformed rectangular domain. For a given boundary condition, the solution to internal points is improved by a number of sweeps; the error on the free-surface boundary condition is then evaluated; subsequently, the solution on the free boundary is updated and the procedure is repeated until convergence. Using this method, Dalrymple \& Cox (1976) showed the influence of the vorticity on wavelength and crest elevation of the wave, considering that the currents vary as trigonometric and hyperbolic sine and cosine functions of the depth. Thomas (1990) performed experimental studies for a finite-amplitude wave train interacting with a steady current containing an arbitrary distribution of vorticity, and moderate agreement was found between the measurements 
and the results obtained using the model of Dalrymple (1977). Ko \& Strauss (2008b) presented a numerical continuation method to find large-amplitude 2-D periodic steady water waves with arbitrary vorticity, which was used for discussing the relationship between amplitude, hydraulic head, depth and mass flux for variable vorticity cases (Ko \& Strauss 2008a). This method neither uses truncated Fourier expansion nor makes any assumption on the water depth or wave amplitude. Further numerical analyses of water waves on rotational flows with discontinuous vorticity can be found in Strauss (2010), Constantin \& Strauss (2011) and Constantin (2012a). The ethos of the approaches towards wave computation is that the local bifurcation from a laminar flow to a small-amplitude wave solution is predicted by a dispersion relation, the algorithm beginning with the linearized solution. The wave solution is then continued with increasing wave height until the solution becomes close to a wave in a flow with stagnation point (global bifurcation). The dispersion relation has been derived for small-amplitude periodic water waves travelling on rotational flows for various vorticity cases, e.g. see Constantin \& Strauss (2011) and Constantin (2012a) and Martin (2015) for flows with two layers. Karageorgis (2012) discussed dispersion relations for several types of non-constant vorticities. High-order approximations for the constant vorticity case were discussed in Constantin, Kalimeris \& Scherzer (2015a). Amann \& Kalimeris (2018) expanded upon the method of Ko \& Strauss (2008b), by allowing non-uniform grid points and enabling the continuation with vorticity as a bifurcation parameter. Using the latter scheme they successfully discovered a new part of the bifurcation curve for some critical constant vorticity values (see also Kalimeris 2018). Furthermore, Constantin, Kalimeris \& Scherzer (2015b) proposed a penalization method to solve for large-amplitude waves. Note that using the method of Ko \& Strauss (2008b), the mean water depth is recovered from the solution, which leads to a small variation in the value of mean water depth along the bifurcation curve (Ko \& Strauss 2008a). The mean depth can be held constant by following the reformulation proposed by Henry $(2013 a, b)$.

Considerable understanding of water waves on irrotational flows and flows with constant vorticity has been established recently. For irrotational flows, Constantin (2006) proved analytically that the particle trajectories are not closed on Stokes waves and Henry (2006) extended the proof to deep water. Constantin \& Villari (2008) and Matioc (2010) showed that there are no closed particle orbits in linear periodic water waves. Borluk \& Kalisch (2012) reconstructed the velocity fields and the particle paths of long-crested waves on irrotational flows using the Korteweg-de Vries (KdV) approximation. These particle paths were found to be consistent with the Stokes drift but with increasing amplitude. Carter, Curtis \& Kalisch (2020) studied the particle paths in the nonlinear Schrödinger models. Grue, Kolaas \& Jensen (2014) experimentally studied the velocity field below large-amplitude periodic water waves and the results are generally consistent with nonlinear theories. Grue \& Kolaas (2017) further investigated the particle paths in steep waves at finite water depth (without background current) experimentally, focusing on the Stokes drift. It was shown that for steep waves on irrotational flow, two closed particle paths occur at two vertical positions, in contrast to the theoretical results (Constantin 2006). The lower closed particle paths occur at a position quite close to the bed due to the boundary layer effect, whereas the theoretical study by Constantin (2006) assumes inviscid water. It is also clear from Grue \& Kolaas (2017) that the thickness of the boundary layer is approximately 5 per cent of the water depth, which is significantly affected by viscosity effect. Paprota \& Sulisz (2018) developed a theoretical model for studying the particle paths of nonlinear waves generated in a closed flume and the Stokes drift velocity obtained from experimental data (Paprota, Sulisz \& Reda 2016) were found to be quite consistent with theories. For a summary of the present analytical 


\section{Chen, B. Basu and C.-I. Martin}

knowledge on the properties of irrotational flows, including the velocity field, pressure and particle trajectories, see Constantin (2015). For waves on a linear-shear flow, Ehrnström (2008) studied the pattern of streamlines and particle trajectories and pointed out that for non-positive vorticity the particles display a forward drift. Using an asymptotic expansion for the streamfunction, Ali \& Kalisch (2013) constructed the pressure beneath steady long gravity water waves travelling on flows with constant background vorticity. Curtis, Carter \& Kalisch (2018) investigated the effect of linear background shear on the properties of wave trains, particularly on particle paths, in deep water using nonlinear Schrödinger models. Numerically, for understanding the particle paths or trajectories, Nachbin \& Ribeiro-Junior (2014) proposed a method for particle trajectories in Stokes waves in the boundary integral formulation for irrotational and constant vorticity cases. A summary of the flow structure beneath rotational flows with constant vorticity and the corresponding numerical method can be found in Nachbin \& Ribeiro-Junior (2017). Lately, Dyachenko $\&$ Hur $(2019 a)$ recovered various characteristics of wave profiles in flows with constant vorticity.

For rotational flow with general vorticity but subject to certain constraints, Constantin \& Strauss (2007) studied the points of maximal horizontal velocity of waves near stagnation. Further understanding on the velocity field has been established by Varvaruca (2008) and Basu (2019). Vasan \& Oliveras (2014) discussed the pressure beneath rotational flows with constant vorticity pointing out interesting applications in wave profile recovery (Oliveras et al. 2012; Basu 2017, 2018).

For surface waves on rotational flows, there are still a number of open problems yet to be solved (Strauss 2012). However, it becomes extremely difficult to capture the properties of the surface wave and the flow structure analytically for complex cases. As manifested in the literature, numerical investigations can provide us with important insights for such cases. To deal with the otherwise intractable problem of rotational flows with arbitrary vorticity distribution, contrary to the widely assumed linear-shear flows, we focus on the shear flow with piece-wise constant vorticity which is the simplest case deviating from constant vorticity. This is a valid approximation for waves travelling on depth-varying currents (Dalrymple 1974b; Swan et al. 2001). In particular, we are concerned with shear flows with two layers. It is known that wind typically induces vorticity in the layer near the surface, while near the ocean shore, vorticity can be generated at the bottom, e.g. by tidal action (Ko \& Strauss 2008a). In this study, we report on a new branch of the bifurcation curve for waves on rotational flows with discontinuous vorticity and investigate the pressure beneath the waves for flows with nearly approaching stagnation points. Further, we develop a method for computing particle paths based on the exact velocity field solved numerically. The particle paths are of increasing interest since they are related to the Lagrangian transport and the Stokes drift in ocean (Van den Bremer \& Taylor 2016; Van den Bremer \& Breivik 2017).

The rest of this paper is structured as follows. In $\S \S 2$ and 3 , we briefly introduce the problem and the numerical methods respectively. The numerical results are then presented in $\S 4$. We close the study by a brief summary in $\S 5$.

\section{Statement of the problem}

We consider 2-D steady periodic rotational gravity travelling surface waves, of period $2 L$ ( $L>0$ being a constant) propagating over water flows bounded below by the flat bed $y=-d$, where $d>0$ is a constant and $y$ is the vertical coordinate with $y=0$ placed at the mean water level. The water is assumed to be inviscid. In the numerical investigation, we shall place our focus on the case with discontinuous vorticity of piece-wise constant type. 


\section{Rotational flows beneath periodic waves near stagnation}

\subsection{Governing equations}

The time-dependent water wave problem is presented in the Cartesian $(X, Y)$-coordinate system with the origin on the mean water level, having the $X$-axis pointing in the horizontal direction and the $Y$-axis pointing upwards. The steady character of the problem we consider allows us to repeal the time dependence by using the change of coordinates $(X-c t, Y) \mapsto(x, y)$, where $c$ is the constant speed of the travelling waves and $t$ denotes time. Hereafter, we will be working in the moving frame $(x, y)$ with the origin placed under the crest, except for the computation of particle paths. In this setting, the motion of the water flow is governed by the Euler (or momentum) equations, the equation of mass conservation supplemented by the kinematic boundary conditions on the two boundaries of the flow and the dynamic boundary condition on the free surface. The latter condition at the free surface decouples the motion of the water from the motion of the air above, while the former kinematic conditions convey the fact that the bed and the free surface are impermeable boundaries, cf. Constantin (2011).

Aiming at a simplification of the analysis we introduce the streamfunction, denoted by $\psi$, and defined (up to a constant) by

$$
\psi_{y}=u-c \text { and } \psi_{x}=-v \text {. }
$$

Here, $(u, v)$ is the velocity field in the $(x, y)$-coordinates. The mass flux relative to the uniform flow at speed $c$ (normalized with respect to density) is

$$
p_{0}=\int_{-d}^{\eta(x)}[u(x, y)-c] \mathrm{d} y,
$$

further referred to as the relative mass flux. It turns out from the mass conservation equation and from the kinematic boundary condition on the surface (denoted by $y=\eta(x)$ ) that $p_{0}$ is a constant.

From $(2.1 a, b)$ and the kinematic condition on the free surface we infer that $\psi$ is constant on the free surface. Therefore, we choose $\psi=0$ on the surface and thus, by (2.2), we have $\psi=-p_{0}$ at the bottom $y=-d$. From the first relation in $(2.1 a, b)$, we have

$$
\psi(x, y)=-p_{0}+\int_{-d}^{y}[u(x, s)-c] \mathrm{d} s,
$$

where $s$ is the variable of integration. Since we assume that $u<c$ throughout the fluid (Constantin \& Strauss 2004), $p_{0}$ is always negative. Following Constantin \& Strauss (2004), the fluid vorticity is defined as

$$
\omega:=u_{y}-v_{x} .
$$

Moreover, the assumption of no-stagnation (since $u<c$ ) implies, cf. Constantin (2011), that there is a function of one variable, which we denote by $\gamma$, such that $\omega(x, y)=$ $\gamma(\psi(x, y))$ for all $(x, y)$ in the water domain. Also from formula $(2.1 a, b)$, we have that $\omega=\Delta \psi$. Hence, the fluid vorticity satisfies

$$
\Delta \psi=u_{y}-v_{x}=\gamma(\psi) .
$$

Here, $\gamma$ is the function already found by the previous argument involving the no-stagnation condition, as presented in the book by Constantin (2011).

The density normalized hydraulic head $E$ is expressed as

$$
E=\frac{1}{2}\left[(u-c)^{2}+v^{2}\right]+P+g y-\int_{0}^{\psi} \gamma(s) \mathrm{d} s
$$

which is a constant owing to conservation of energy. In the expression, $g$ is gravitational acceleration, $P$ is the pressure (normalized with respect to density) in the fluid and at the 


\section{Chen, B. Basu and C.-I. Martin}

surface $P=P_{\text {atm }}$, the atmospheric pressure which also normalized with respect to density. Therefore, for a given water depth, we can introduce another constant $Q=2\left(E-P_{a t m}+\right.$ $\mathrm{gd}$ ), and the boundary condition on the surface is given by

$$
\psi_{x}^{2}+\psi_{y}^{2}+2 \mathrm{~g}(y+d)=Q \quad \text { on } \psi=0 .
$$

Eventually, we obtain the fixed domain boundary-value problem from the free-surface wave problem by applying the following DJ transformation, i.e.

$$
q=x \quad \text { and } \quad p=-\psi(x, y)
$$

to the relations (2.5) and (2.7), and including the boundary condition at $p=p_{0}$. The equations are

$$
\left.\begin{array}{ll}
\left(1+h_{q}^{2}\right) h_{p p}-2 h_{p} h_{q} h_{q p}+h_{p}^{2} h_{q q}-\gamma(-p) h_{p}^{3}=0 & \text { in } R \\
1+h_{q}^{2}+(2 \mathrm{~g} h-Q) h_{p}^{2}=0 & \text { on } p=0 \\
h=0 & \text { on } p=p_{0}
\end{array}\right\}
$$

where $h(q, p)=y+d$ is the height function and $R$ is the rectangle defined by $R=\{(q, p)$ : $\left.-L<q<L, p_{0}<p<0\right\}$.

\subsection{Recovery of wave properties}

We will solve the problem (2.9) for the height function using a numerical method that will be introduced in the next section. For characterizing the waves, we shall recall the recovery of wave properties from the height function. Since in this formulation, the mean water depth $d$ is not fixed, it needs to be recovered first from $h$ by

$$
d=\frac{1}{2 L} \int_{-L}^{L} h(q, 0) \mathrm{d} q .
$$

Afterwards, the water profile is determined by

$$
y=h-d
$$

The wave height $a$ is obtained as

$$
a=h(0,0)-h(L, 0)
$$

as we are concerned with waves of one crest and one trough per period. Further, the velocity field is recovered by

$$
(c-u, v)=\left(\frac{1}{h_{p}},-\frac{h_{q}}{h_{p}}\right),
$$

recalling the DJ transformation (see Constantin (2011) for details). The pressure in the fluid domain can be obtained from the relation (2.6) and here we define a relative pressure, 
$\tilde{P}=P-P_{a t m}$, for the following discussions as

$$
\begin{aligned}
\tilde{P}= & -\frac{1+h_{q}^{2}}{2 h_{p}^{2}}-\mathrm{g} h+\frac{Q}{2}-\int_{0}^{p} \gamma(-s) \mathrm{d} s=-\frac{\left[(u-c)^{2}+v^{2}\right]}{2}-\mathrm{g}(y+d) \\
& +\frac{Q}{2}-\int_{0}^{p} \gamma(-s) \mathrm{d} s .
\end{aligned}
$$

For computing water particle paths or trajectories, the travelling speed of the wave is recovered (Constantin 2011) by using

$$
c=\kappa-\frac{1}{L} \int_{0}^{L}[u(x,-d)-c] \mathrm{d} x,
$$

where $\kappa$ is the average horizontal current strength on the bed. We then need to solve a set of differential equations (Constantin \& Strauss 2010; Nachbin \& Ribeiro-Junior 2014)

$$
\left.\begin{array}{l}
X^{\prime}=u(X-c t, Y), \\
Y^{\prime}=v(X-c t, Y), \\
(X(0), Y(0))=\left(X_{0}, Y_{0}\right),
\end{array}\right\}
$$

where the prime denotes the derivative with respect to time. Here, we use a numerical integration method to compute the particle paths once the velocity field is solved, as will be detailed in the next section.

\section{Numerical methods}

In this section, we briefly introduce the numerical methods to solve (2.9), for performing numerical continuation to arrive at the solution of waves in flows with closely approaching stagnation points, and for computing the particle trajectories from the velocity field in the Eulerian setting. The dispersion relations and the linearized solutions for local bifurcation from the laminar flow solutions to small-amplitude wave solutions are also recalled for the sake of completeness.

\subsection{Discretization and implementation}

Following the methods in Ko \& Strauss (2008b) and Amann \& Kalimeris (2018), we use a finite difference scheme to discretize the computational domain. Taking advantage of the symmetry of $h$ function with respect to $q=0$ (Constantin, Ehrnström \& Wahlén 2007), we set the computational domain as a fixed rectangle $[0, L] \times\left[p_{0}, 0\right]$ to improve the computation efficiency. We first discretize the domain using uniformly distributed grid points and then refine the grid size near the surface and the bottom under the crest $(q=0)$. In the case of discontinuous vorticity, we additionally refine the grid size near the location where the vorticity changes abruptly. We apply the central difference scheme at interior grid points, and at left and right boundary points by mirroring $h$ along $q=0$ and $q=L$, considering the periodicity and symmetry properties of the waves in absence of stagnation points. At the boundaries $p=0$ and $p=p_{0}$, we apply the backward and forward difference schemes, respectively. Note that the refinement is achieved by reducing the grid size smoothly so that the central difference still has a second-order accuracy, approximately. 


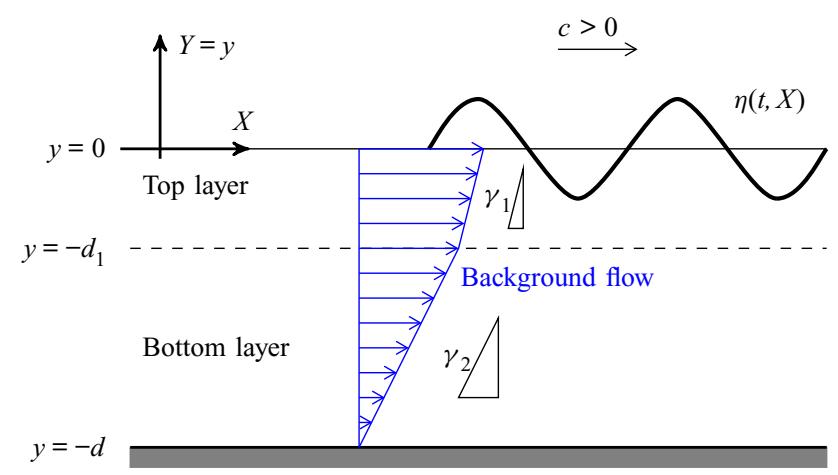

Figure 1. The setting of nonlinear periodic waves travelling on rotational flow with discontinuous vorticity. In the figure, $\gamma_{1}$ and $\gamma_{2}$ are positive and $d_{1}$ denotes the thickness of the top layer.

For implementation, we use the open-source library Eigen (Guennebaud \& Jacob 2010) for solving the nonlinear algebraic equations subsequent to the finite difference discretization. We use the predictor-corrector method detailed in Amann \& Kalimeris (2018) for numerical continuation and write our own code on the basis of Eigen. In the following numerical analysis, we discretize the domain first by an equidistant $250 \times 750$ grid with additional refinement at locations where the stagnation point may occur. Following the refinement, the smallest grid size achieved is $1 / 8$ th of the initial size prior to the mentioned refinement.

\subsection{Dispersion relations and linearization solutions for local bifurcation}

As previously mentioned, we focus on the discontinuous vorticity. The vorticity function is expressed as

$$
\gamma= \begin{cases}\gamma_{1}, & p \in\left(p_{1}, 0\right), \\ \gamma_{2}, & p \in\left(p_{0}, p_{1}\right),\end{cases}
$$

where $p_{1}$ corresponds to the value of the streamline at the interface $y=y_{1}$, and $\gamma_{1}$ and $\gamma_{2}$ are the constant vorticity values of the surface layer and the layer adjacent to the bed. The setting of the problem is illustrated in figure 1 . For the case of $\gamma_{1}=\gamma_{2}=0$, the problem reduces to irrotational flow, and the dispersion relation and the linearized solutions are available in Constantin (2011). For the situation of a rotational flow over an irrotational and vice versa, the dispersion relations are explicitly derived in Constantin \& Strauss (2011) and Constantin (2012a), respectively. For steady water waves on two rotational layers, see Martin (2015).

The dispersion relations are nonlinear equations. Here, we solve them using the Newton method. For large vorticity, we apply a simple continuation scheme by using the solution for smaller vorticity for initialization (Seydel 2009).

\subsection{Computation of the particle trajectories}

We numerically compute the particle paths in the physical domain, i.e. in the fixed $(X, Y)$-coordinate. For a particle located at $(X(t), Y(t))$ at time $t$, its velocity is interpolated from the velocities at the neighbouring four grid points (following appropriate translation) using a similar interpolation method as in Umeyama (2012). The interpolation scheme is illustrated in figure 2. Note that in our case, the cell is of trapezoidal shape in the 


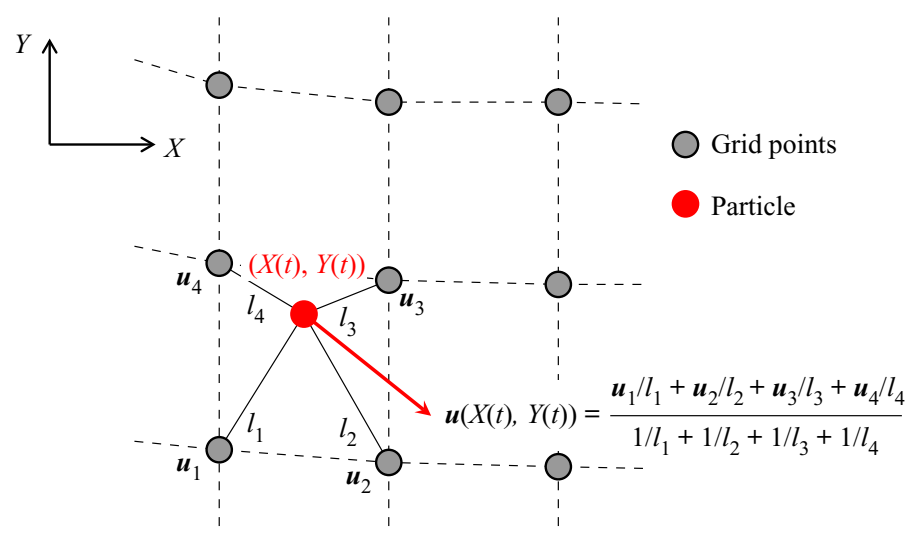

Figure 2. Interpolation strategy for instantaneous particle velocity. In the figure, $u=\left[\begin{array}{ll}u & v\end{array}\right]^{\top}$ is the velocity vector; $l_{1}, l_{2}, l_{3}$ and $l_{4}$ are respective distances of the particle to the four neighbouring grid points.

physical domain. Subsequently, we use numerical integration to solve the first-order differential equations (2.16). This simple interpolation scheme is found to be sufficiently accurate.

\section{Results}

Following Ko \& Strauss $(2008 a, b)$ and Amann \& Kalimeris (2018), we assign $g=9.8$, $2 L=2 \pi$ and $p_{0}=-2$. We focus on the new part of the bifurcation curves, pressure distribution and particle paths under flows with nearly approaching stagnation points. Note that in the present system all the variables are expressed in SI units and a physical system of a different wavelength can be scaled to the $2 \pi$-periodic system analysed in this paper by scaling $g$ and $\gamma$ using the wave number (Constantin 2011). However, the scaling has no influence on the laminar flow solution. In the following, as will be seen, the current velocity at the crest (under zero-amplitude waves) in most of the cases is less than $2.5 \mathrm{~m}$ $\mathrm{s}^{-1}$, which is physically realistic in oceans.

\subsection{Critical vorticity and the new part of the branch of bifurcation diagram}

For the case of constant vorticity, it is known that when the vorticity is below a critical value $\gamma_{\text {crit }}$, the stagnation point first appears at the bottom and at that stage numerical continuation breaks down (Ko \& Strauss 2008b). However, Amann \& Kalimeris (2018) and Kalimeris (2018) found that when $\gamma<\gamma_{\text {crit }}$ but is very close to $\gamma_{\text {crit }}$, the branch of the bifurcation diagram $(a-Q$ plot) has another part which is bounded below and above by two values of the bifurcation parameter $Q$. These bounds correspond to two different waves in flows with stagnation point at the bottom and at the surface. On the new part of the branch, the wave height is further increased, and it is thus referred to as the upper part. To reach this upper part of the solutions, we need to overcome the gap of the bifurcation curve corresponding to waves in flows with stagnation points. For this purpose, Amann \& Kalimeris (2018) resorted to the $\gamma$-continuation scheme where a solution on the bifurcation diagram corresponding to $\gamma_{0}\left(\gamma_{0}>\gamma_{\text {crit }}>\gamma\right)$ is used to initialize local bifurcation to arrive at one solution on the upper part corresponding to $\gamma$. Once one solution is obtained, the continuation in the solution set corresponding to $\gamma$ can be performed again with $Q$ or the wave height as the bifurcation parameter. In the following, we apply this scheme when needed. 

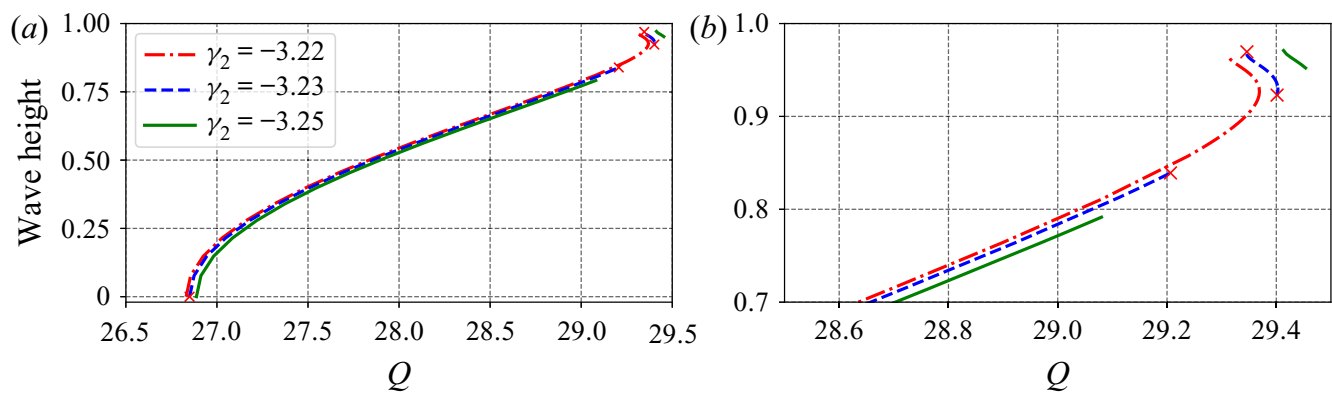

Figure 3. Bifurcation diagrams for cases with $p_{1}=-0.5, \gamma_{1}=0$ (at the top layer) and various different values of $\gamma_{2}$ (at the bottom layer): (a) full diagrams; and $(b)$ a close-up view of the upper parts.

Proceeding from the observations of Amann \& Kalimeris (2018), we investigated the case of discontinuous vorticity and found that the upper part also exists. Furthermore, for the case of discontinuous vorticity, the upper bound on this part always corresponds to waves in flows with the stagnation point at the surface, while its lower bound corresponds to waves in flows with stagnation point either at the bottom or at an internal point where vorticity jump occurs. We first show an example of the former situation. An irrotational flow $\left(\gamma_{1}=0, p_{1}=-0.5\right)$ was considered on a rotational flow with vorticity $\gamma_{2}$. We varied $\gamma_{2}$ to find out the critical value $\gamma_{2}$,crit below which the stagnation point first occurs at the bottom. It was found that $\gamma_{2, \text { crit }}$ is slightly less than -3.22 . Note that with the vorticity values $\gamma_{1}=0$ and $\gamma_{2}=-3.23$ and hence a shearing laminar flow with profile shown in figure 4(a) over a total water depth of $0.787 \mathrm{~m}$, we have the surface velocity, $u=-2.07$ $\mathrm{m} \mathrm{s}^{-1}$ (the current flow direction is opposite to the wave propagation direction). Equation (2.5) with a bifurcation value of $Q=26.85$ (see figure $3 a$ ) leads to the relative surface velocity with respect to the wave speed as $c-u=3.38 \mathrm{~m} \mathrm{~s}^{-1}$, since $u<c$. The wave speed $c$ is $1.31 \mathrm{~m} \mathrm{~s}^{-1}$ as also verified by the dispersion relation for this case. In the case of positive vorticity in the bottom layer, e.g. $\gamma_{1}=0$ and $\gamma_{2}=3$, the water depth is $0.74 \mathrm{~m}$, the current velocity at the surface is approximately $1.53 \mathrm{~m} \mathrm{~s}^{-1}$ and at the local bifurcation the wave speed is $c=3.69 \mathrm{~m} \mathrm{~s}^{-1}$. These values of the physical parameters indicate that the settings considered in this paper are realistic.

We then further decreased $\gamma_{2}$ and found the upper part, as shown in figure 3 . For the case of $\gamma_{2}=-3.23$, the streamlines of wave solutions at the four endpoints (marked with crosses in figure 3 ) of the two parts of the bifurcation curve are shown in figure 4 . Figure 4 clearly shows that the stagnation point first occurs at the bottom and then at the crest. Figure 5 shows the horizontal velocities $c-u$ at the bottom and at the surface under the crest along the bifurcation curves. Note that both waves in figures $4(b)$ and $4(c)$ correspond to flows close to having a stagnation point at the bottom, and the horizontal velocities $c-u$ at the bottom in these two cases are comparable, as in figure 5(a), while the wave in figure $4(c)$ has a larger height, a sharper crest and smaller horizontal velocity $c-u$ at the surface (see figure $5 b$ ). It is seen from figure 5 that the velocity $c-u$ at the bottom is first decreasing and then increasing along the bifurcation curve, with the presence of a gap for $\gamma_{2}<\gamma_{2}$,crit, while the velocity $c-u$ at the crest is decreasing monotonically. Indeed, at the uppermost end of the bifurcation diagram in all those three cases, the velocities $c-u$ at the surface are still larger than those at the bottom, the stagnation point is expected to occur at the crest because the velocity at the surface is decreasing while at the bottom is increasing with respect to the increasing wave height. 

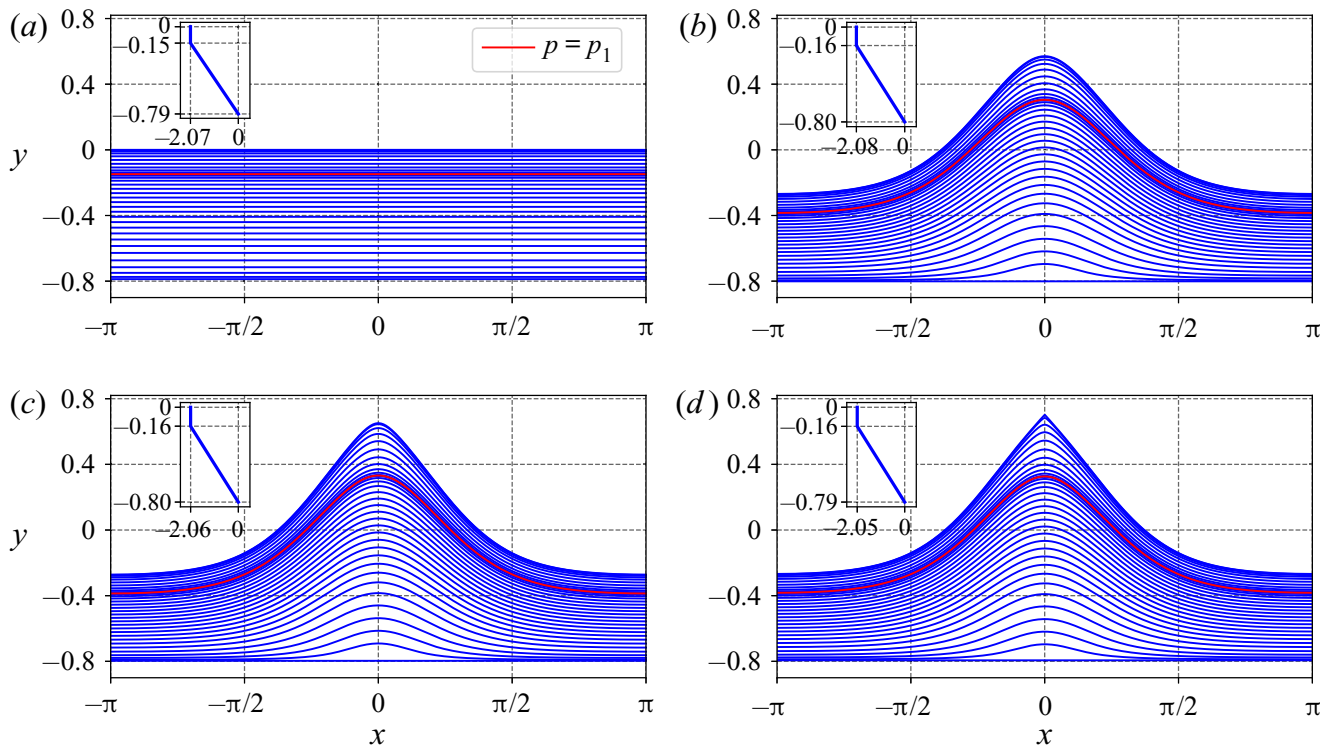

Figure 4. Streamlines of flows at the endpoints of each of the two parts of the bifurcation curve corresponding to $p_{1}=-0.5, \gamma_{1}=0$ (at the top layer), and $\gamma_{2}=-3.23$ (at the bottom layer): $(a)$ laminar flow; $(b)$ wave at the upper bound of the lower part; $(c)$ wave at the lower bound of the upper part; and $(d)$ wave at the upper bound of the upper part. In the figures, the insets show the background current profile when there is no wave motion.
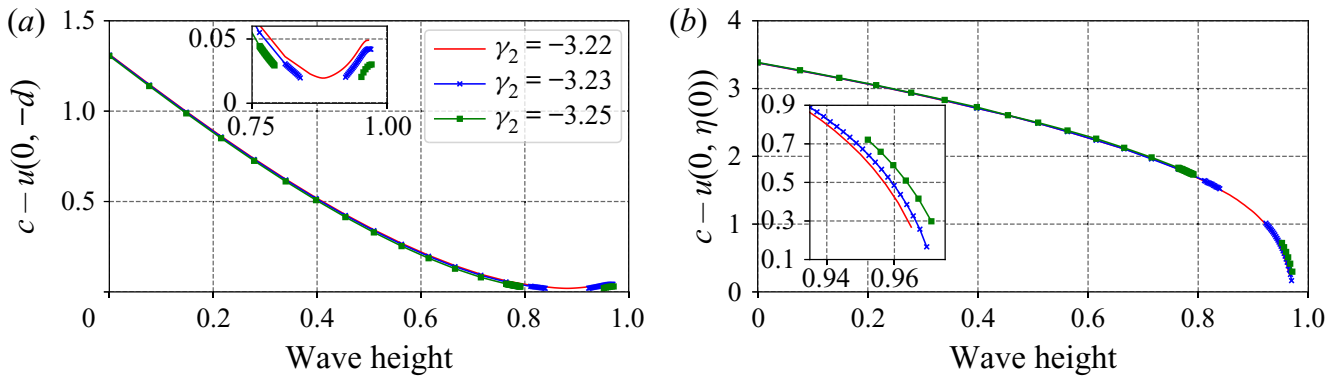

Figure 5. Horizontal velocity, $c-u$, under the crest along the bifurcation curve for $\gamma_{1}=0$ (at the top layer), $p_{1}=-0.5$, and various different values of $\gamma_{2}$ (at the bottom layer): $(a)$ at the bottom and $(b)$ at the surface. In the figures, the insets are close-up views of the curves when approaching the largest wave height.

We further consider a layer $\left(p_{1}=-0.7\right)$ of rotational flow with vorticity $\gamma_{1}$ on an irrotational flow $\left(\gamma_{2}=0\right)$, following the setting in Ko \& Strauss $(2008 a)$ and Constantin $\&$ Strauss (2011). We found that the critical vorticity $\gamma_{1, \text { crit }}$ is slightly below -10.41 (a value refined from -10 in Ko \& Strauss 2008a). By using $\gamma_{1}$ as the bifurcation parameter, we also found the upper part of the branch of the bifurcation diagram when $\gamma_{1}$ is less but very close to $\gamma_{1, \text { crit }}$. Figure 6 shows the bifurcation diagrams for $\gamma_{1}=-10.41,-10.42$ and -10.45 . For $\gamma_{1}=-10.42$, the streamlines of the waves corresponding to the four endpoints of the bifurcation curve are shown in figure 7 , which clearly illustrates that the stagnation point first occurs internally and later occurs at the crest with further increased wave height. Figure 8 shows the variation of the velocities $c-u$ at $p=p_{1}$ and at the surface along the bifurcation curves. The velocity $c-u$ at the position where vorticity jumps, is first decreasing and then increasing with respect to the wave height. Notably, the 


\section{Chen, B. Basu and C.-I. Martin}
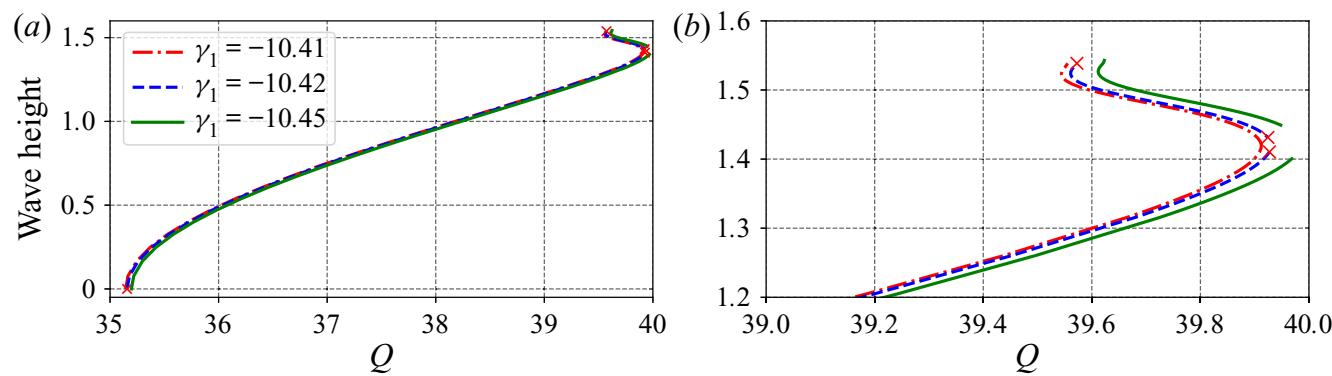

Figure 6. Bifurcation diagrams for cases with $p_{1}=-0.7, \gamma_{2}=0$ (at the bottom layer) and various different values of $\gamma_{1}$ (at the top layer): ( $a$ ) full diagrams; and $(b)$ a close-up view of the upper parts.
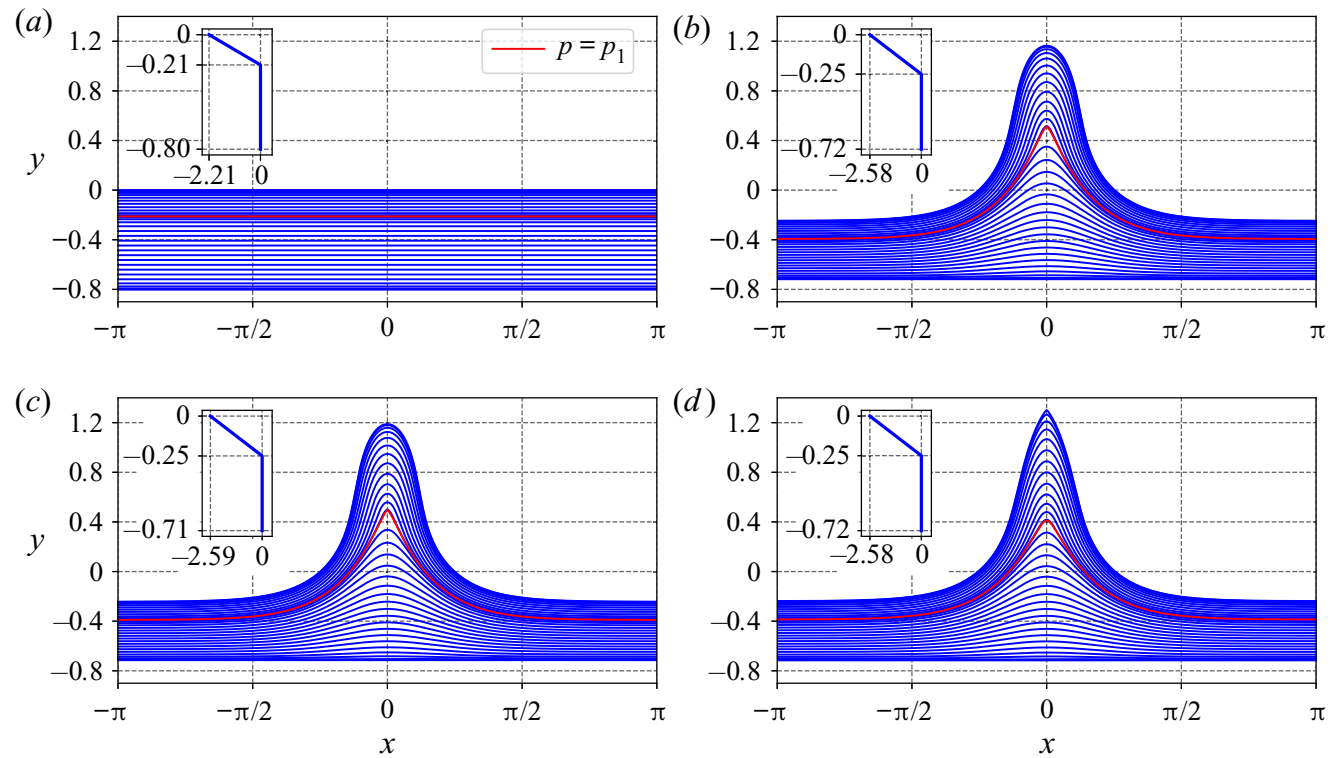

Figure 7. Streamlines of flows at the endpoints of each of the two parts of the bifurcation curve corresponding to $p_{1}=-0.7, \gamma_{1}=-10.42$ (at the top layer), and $\gamma_{2}=0$ (at the bottom layer): $(a)$ laminar flow; $(b)$ wave at the upper bound of the lower part; $(c)$ wave at the lower bound of the upper part; and $(d)$ wave at the upper bound of the upper part. In the figures, the insets show the background current profile when there is no wave motion.

velocity decreases slightly again when close to the wave in flows with stagnation point at the surface. Again, the velocity $c-u$ on the crest is decreasing monotonically along with the increasing wave height.

To summarize, in this subsection, we have observed new features of the bifurcation curves with fixed mass flux, for large-amplitude waves on flows with discontinuous vorticity. Specifically, we show that along the bifurcation curve the stagnation point can first occur at the bottom or internally and then occur again at the surface for some particular vorticity distributions.

\subsection{Pressure distribution}

We now present the pressure distribution for waves with stagnation points at the bottom, at an internal position and at the surface, for the case with discontinuous vorticity. 

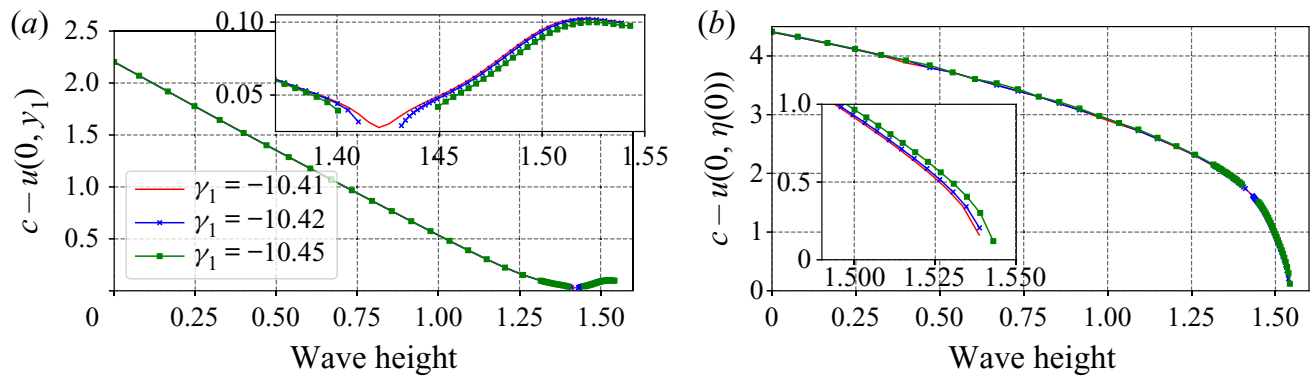

Figure 8. Horizontal velocity, $c-u$, under the crest along the bifurcation curve for $\gamma_{2}=0$ (at the bottom layer), $p_{1}=-0.7$, and varying $\gamma_{1}$ (at the top layer): $(a)$ at the position where vorticity jumps $\left(y=y_{1}\right.$ corresponds to $\left.p=p_{1}\right)$ and $(b)$ at the surface. In the figures, the insets are close-up views of the curves when approaching the largest wave height.

(a)

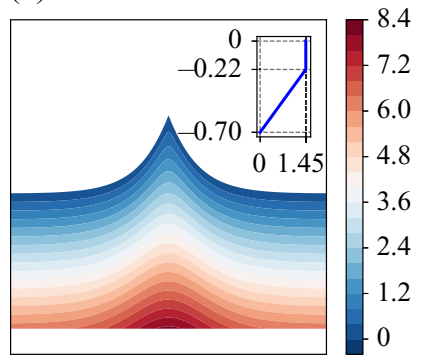

(b)

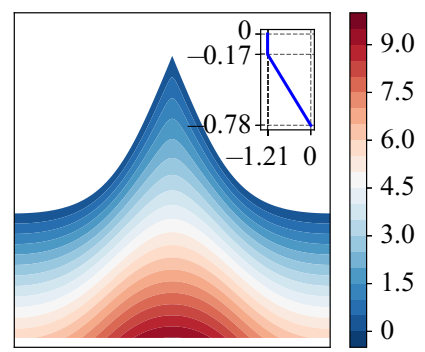

(c)

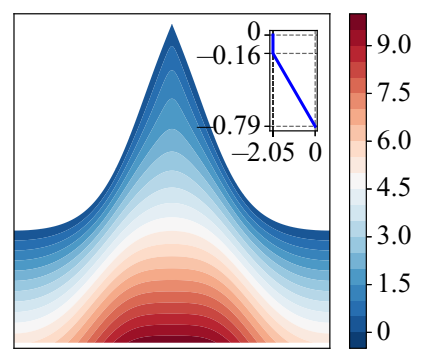

Figure 9. Pressure distribution for waves approaching waves with stagnation point at the crest for $p_{1}=-0.5$, $\gamma_{1}=0$ (at the top layer), and: (a) $\gamma_{2}=3$; (b) $\gamma_{2}=-2$; and (c) $\gamma_{2}=-3.23$. In the figure, the insets show the corresponding background shear flow when there is no wave motion.

For an irrotational flow on a rotational flow with $p_{1}=-0.5$ and $\gamma_{1}=0$, we consider the cases corresponding to $\gamma_{2}=3,-2,-3.23$ and -5 . For waves close to the case of flows having a stagnation point at the crest, the pressure distributions are shown in figure 9, and figure 10 compares the pressure along the bottom and along the crest line. For waves in flows close to having a stagnation point at the bottom, the pressure distribution demonstrated in figure 11, and figure 12 shows the pressure along the bottom and the crest line. Two features of the pressure distributions are consistent with the pressure beneath a Stokes wave as proved by Constantin \& Strauss (2010) and those can be stated as 'the pressure in the fluid strictly decreases horizontally away from a crest line toward its neighbouring trough lines, and the pressure strictly increases with depth'. Even though, when $\gamma_{2}$ is close to the critical value, the pressure along the bottom displays a 'plateau' behaviour, as previously pointed out by Amann \& Kalimeris (2018) for the case of constant vorticity, the pressure is still decreasing away from the crest line, slowly.

For a rotational flow on an irrotational flow, we analyse the cases for $\gamma_{1}=5,-5,-10.42$ and -12 . Figure 13 shows the pressure distribution in flows close to having a stagnation point at the crest, and variations of the pressure along the bottom and along the crest line are illustrated in figure 14. The pressure distribution in flows when internal stagnation points are almost developed is illustrated in figure 15. Correspondingly, figure 16 shows the pressure along the streamline $\psi=-p_{1}$ and along the crest line. From these figures, it is seen that none of the facts regarding the pressure distribution for an irrotational Stokes wave (Constantin \& Strauss 2010) hold, as also observed by 

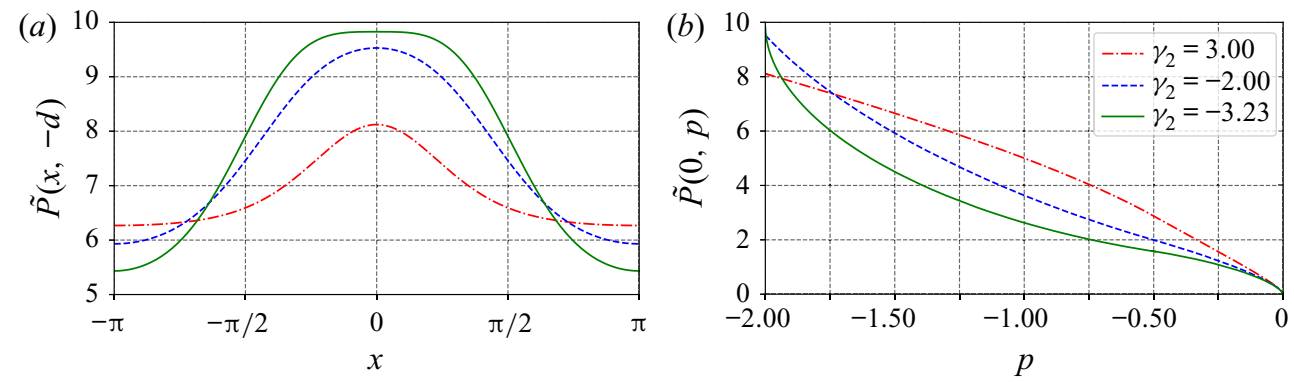

Figure 10. Pressure distribution in flows with an approaching stagnation point at the crest for $p_{1}=-0.5$, $\gamma_{1}=0$ (at the top layer) and various different values of $\gamma_{2}$ along $(a)$ the bottom, and $(b)$ the crest line.

(a)

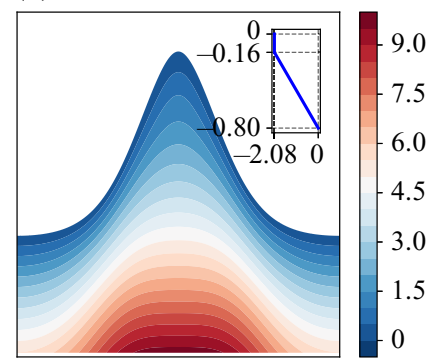

(b)

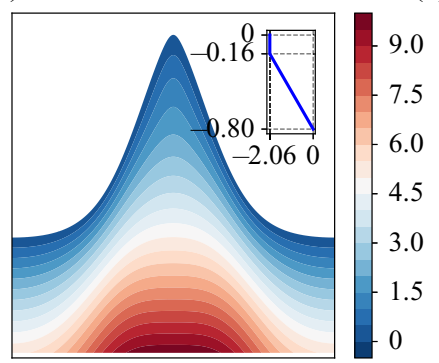

(c)

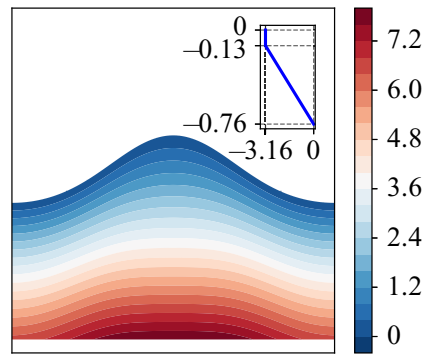

Figure 11. Pressure distribution in flows with an approaching stagnation point at the bottom for $p_{1}=-0.5$, $\gamma_{1}=0$ (at the top layer), and: (a) $\gamma_{2}=-3.23$ (below gap); (b) $\gamma_{2}=-3.23$ (above gap); and (c) $\gamma_{2}=-5$. In the figure, the insets show the corresponding background shear flow when there is no wave motion.
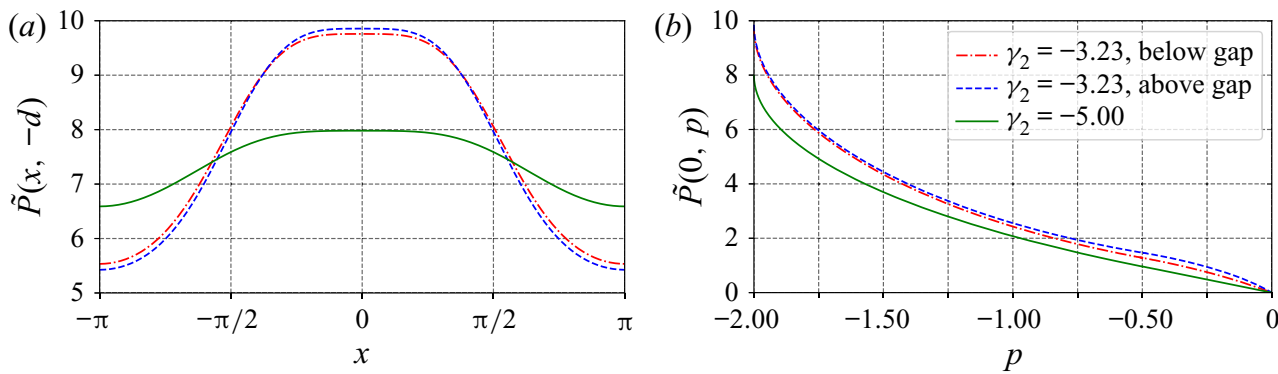

Figure 12. Pressure distribution in flows with an approaching stagnation point at the bottom for $p_{1}=-0.5$, $\gamma_{1}=0$ (at the top layer) and various different values of $\gamma_{2}$ along $(a)$ the bottom, and $(b)$ the crest line.

Ribeiro, Milewski \& Nachbin (2017) for rotational flows with constant vorticity. When the vorticity $\gamma_{1}$ is below a critical value, the pressure along the streamline $\psi=-p_{1}$ achieves a local minimum under the crest, as opposed to the maximum there when $\gamma_{1}$ is large. For smaller $\gamma_{1}$, the relative pressure $\tilde{P}$ at this position becomes negative (i.e. sub-atmospheric), which is the minimum along the streamline. The pressure attaining a value less than the atmospheric pressure has also been observed by Ali \& Kalisch (2013) for the case of constant vorticity using a model based on an asymptotic expansion for the stream functions. When $\gamma_{1}$ is below a critical value, the pressure below but close to the layer where the vorticity jump occurs also exhibits the 'plateau' behaviour, internally, see figure 15. Figures 14-16 show that when $\gamma_{1}$ is much larger than the critical value, the 
(a)

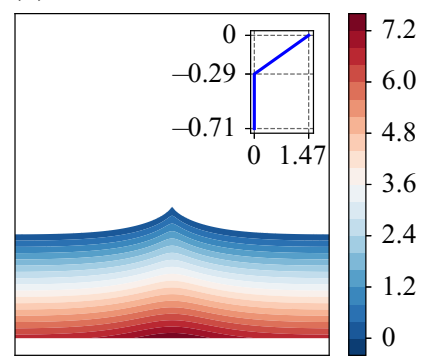

(b)

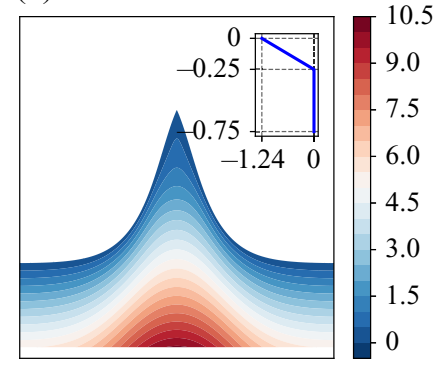

(c)

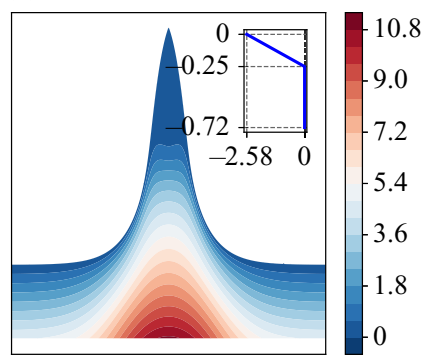

Figure 13. Pressure distribution in flows with an approaching stagnation point at the crest for $p_{1}=-0.7$, $\gamma_{2}=0$ (at the bottom layer), and: $(a) \gamma_{1}=5 ;(b) \gamma_{1}=-5$; and $(c) \gamma_{1}=-10.42$. In the figure, the insets show the corresponding background shear flow when there is no wave motion.
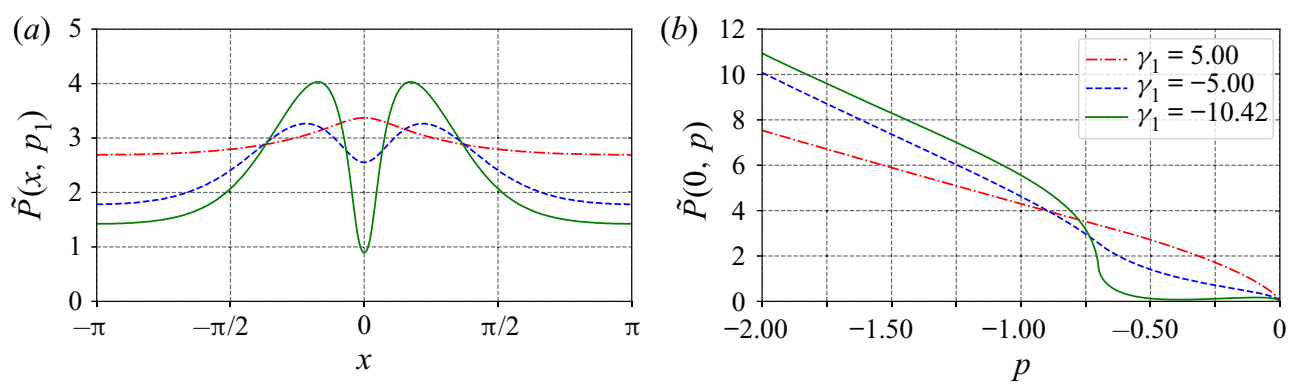

Figure 14. Pressure distribution in flows with an approaching stagnation point at the crest for $p_{1}=-0.7$, $\gamma_{2}=0$ (at the bottom layer) and various different values of $\gamma_{1}$ along $(a)$ the streamline $\psi=-p_{1}$, and $(b)$ the crest line.

pressure is increasing with depth along the crest line. Interestingly, when $\gamma_{1}$ is quite close to $\gamma_{1, \text { crit }}$, the pressure $\tilde{P}$ along the crest line first increases slightly, and then decreases, and increases again when close to the position where the vorticity jump occurs; the overall pressure in the surface layer is close to the atmospheric pressure, see figure 14(b) for $\gamma_{1}=-10.42$. With a further decrease in the value of $\gamma_{1}$ below the critical value, the relative pressure in the surface layer becomes negative, first decreasing from the crest and then increasing near the position where the vorticity jump takes place. To understand the reason for the development of a negative relative pressure $\tilde{P}$ in the surface layer near the crest line, we recall the expression (2.14). For the cases illustrated in figure 14, the term $\int_{0}^{p} \gamma(-s) \mathrm{d} s$ in (2.14) is positive in the surface layer and increases rapidly with depth since $\left|\gamma_{1}\right|$ is large, the term $\mathrm{g}(y+d)$ is also large considering the large wave height, and the velocity $c-u$ is also not small, as shown in figure 17; hence, $\tilde{P}$ in the surface layer becomes negative. Further decreasing $\gamma_{1}$ (with increasing $\left|\gamma_{1}\right|$ ), it seems that the minimal pressure along the crest line will keep decreasing.

\subsection{Particle trajectories}

We proceed by computing the particle trajectories under the waves corresponding to flows with nearly developed stagnation points, for the previously mentioned cases. To recover the wave speed using expression (2.15), we assume that the current strength at the bed is zero, i.e. $\kappa=0$. Note that the choice of a different setting (adding an arbitrary uniform current) may lead to variations of the resulting particle paths. For example, when studying particle paths under waves on linear-shear flow, Ribeiro et al. (2017) set the current strength at the 
(a)

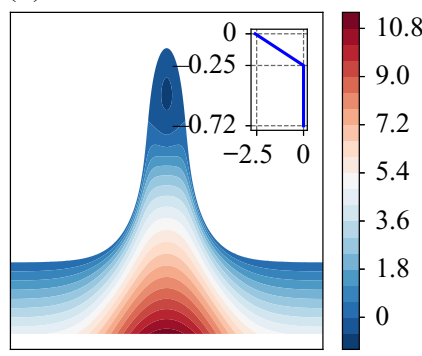

(b)

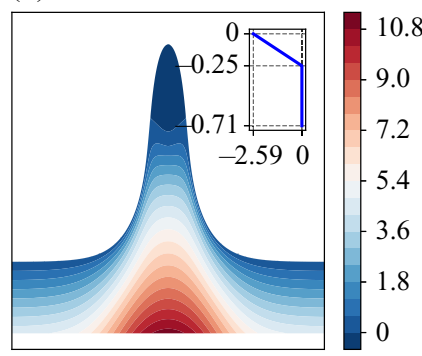

(c)

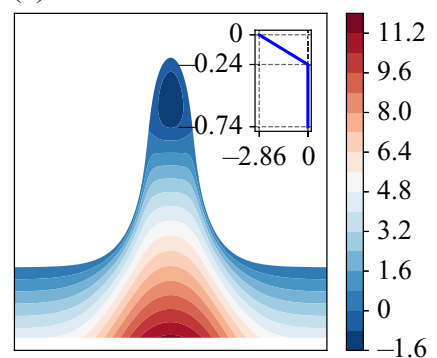

Figure 15. Pressure distribution in flows with an approaching stagnation point at a position where vorticity jump occurs, for $p_{1}=-0.7, \gamma_{2}=0$ (at the bottom layer), and: (a) $\gamma_{1}=-10.42$ (below gap); (b) $\gamma_{1}=-10.42$ (above gap); and $(c) \gamma_{1}=-12$. In the figures, the insets show the background current profile when there is no wave motion.
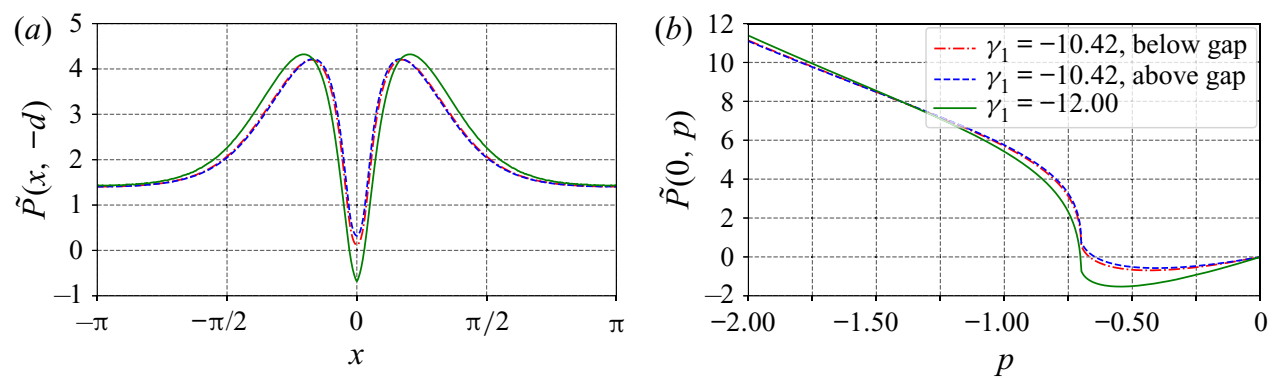

Figure 16. Pressure distribution in flows with an approaching stagnation point at a position where vorticity jump occurs for $p_{1}=-0.7, \gamma_{2}=0$ and various different values of $\gamma_{1}$ along $(a)$ the streamline $\psi=-p_{1}$, and (b) the crest line.

(a)

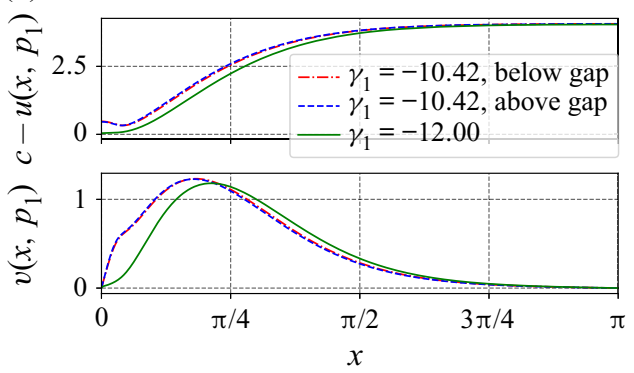

(b)

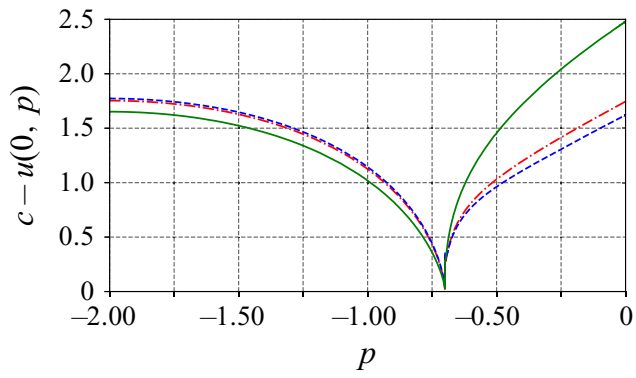

Figure 17. Variation of velocities, $c-u$ and $v$, with $p_{1}=-0.7, \gamma_{2}=0$ (at the bottom layer), and various different values of $\gamma_{1}:(a) c-u$ and $v$ along the streamline $\psi=-p_{1}$, and $(b) c-u$ along the crest line $(v$ is zero along this line and hence not shown).

mid-depth to be zero (to ensure a flow with no net mass flux in the case of zero-amplitude waves). However, we here focus on the relative horizontal displacements of the particles along the depth, which are not affected by the presence of a uniform background current.

In the following computation and illustration of the particle trajectories, we set that: (i) the initial positions of the particles are under a zero-crossing point of the wave profile (Paprota \& Sulisz 2018); (ii) the particles trajectories are plotted for the waves travelling rightwards for two wave periods, i.e. for a time of $4 L / c=4 \pi / c$, and the final position of 

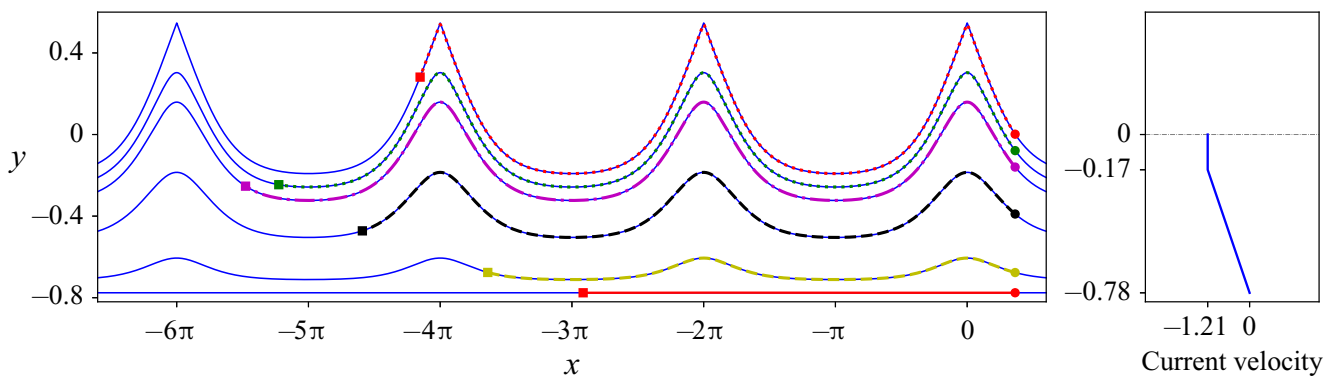

Figure 18. (a) Particle trajectories under the waves in flows close to having a stagnation point at the crest with $p_{1}=-0.5, \gamma_{1}=0$, and $\gamma_{2}=-2$ in the moving frame; $(b)$ the background current profile when there is no wave motion.
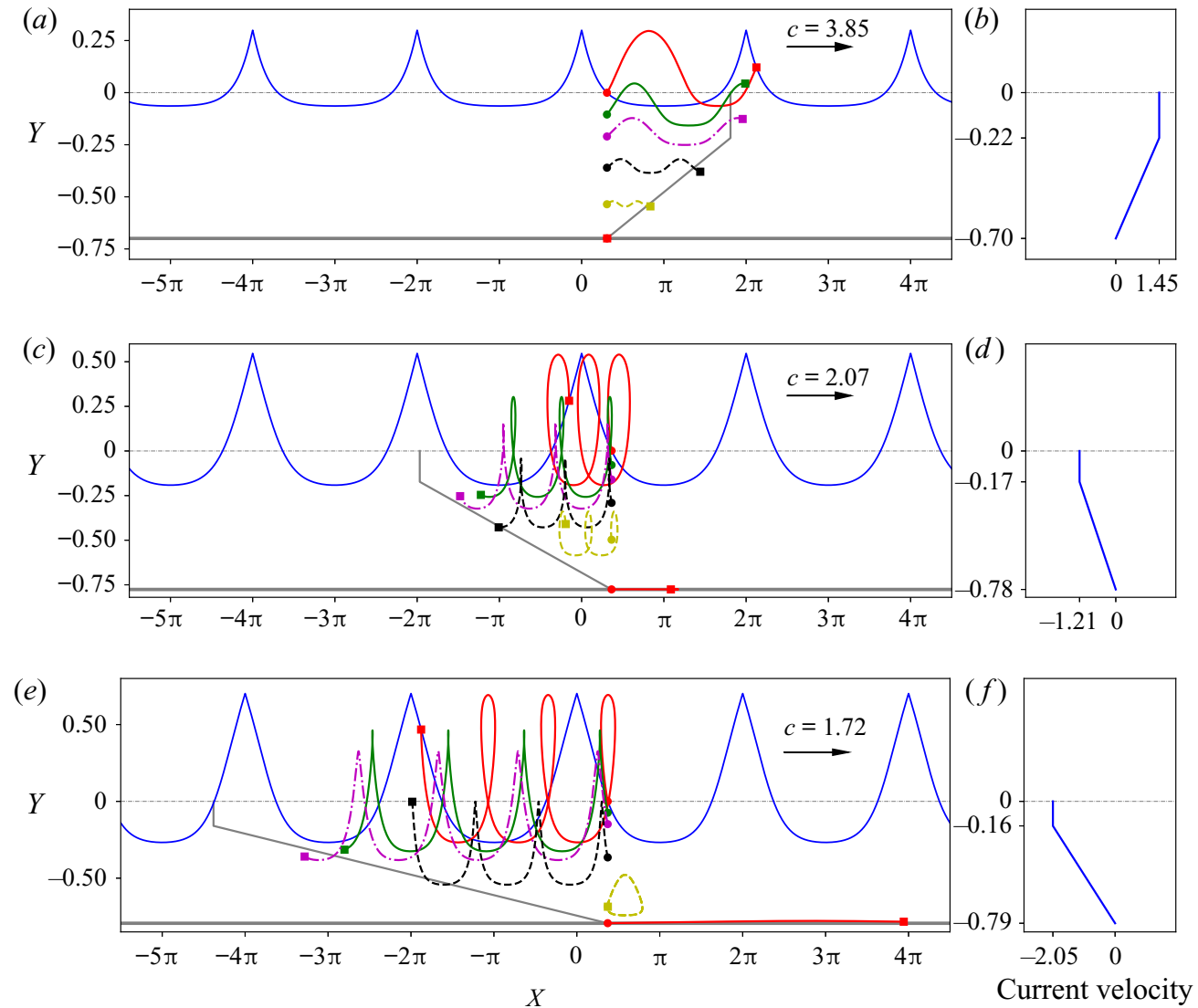

Figure 19. Particle trajectories under the waves in flows close to having a stagnation point near the crest, for $p_{1}=-0.5$ and $\gamma_{1}=0:(a) \gamma_{2}=3 ;(c) \gamma_{2}=-2 ;(e) \gamma_{2}=-3.23$. The background current profiles corresponding to zero-amplitude waves are shown in $(b, d, f)$, respectively. In the plot of the particle paths, the grey lines indicate the final position of the particles if under zero-amplitude waves after the time $4 L / c$.

the particles under zero-amplitude waves are also indicated in the graphs for comparison. In the graphs, six particle trajectories are plotted. Three of those particles are located at the surface, at the interface and at a position very close to the bottom but not exactly at the bottom. The other three particles include one located in the top layer and two located 

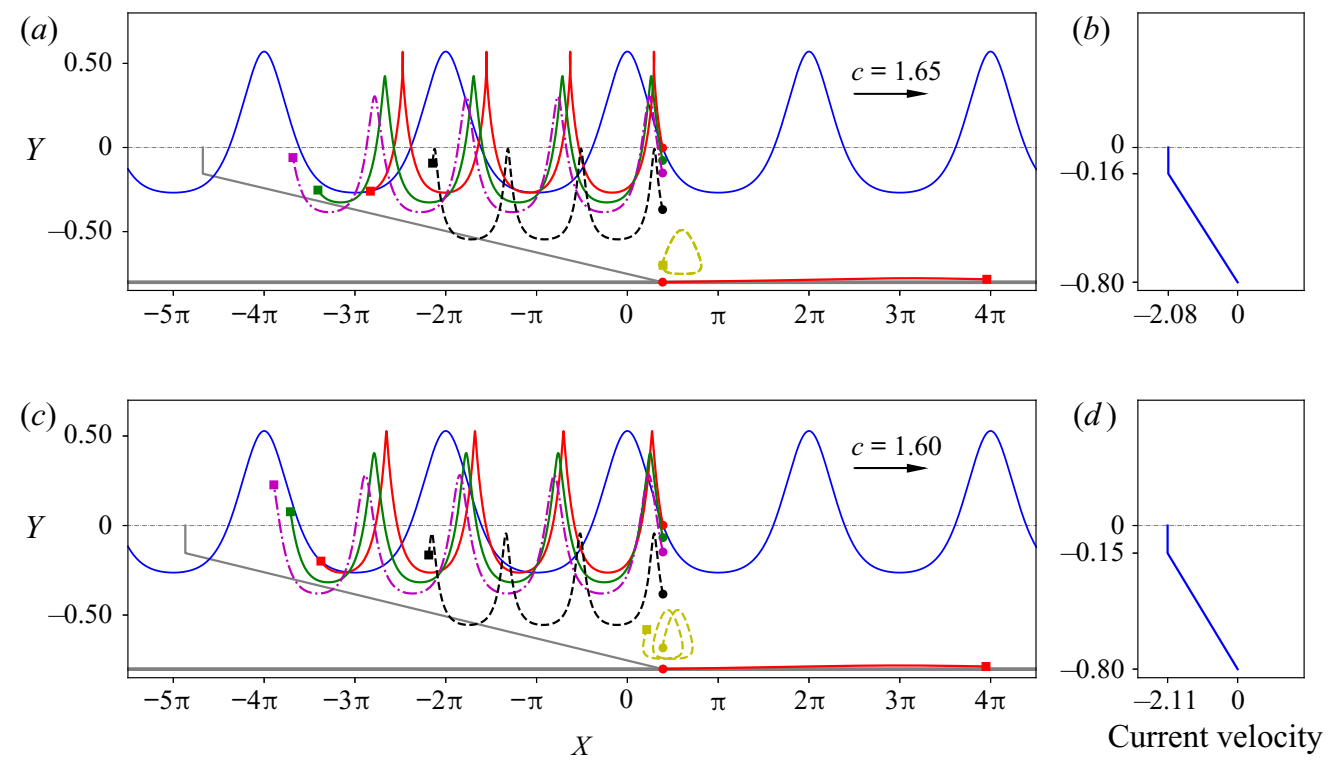

Figure 20. Particle trajectories under the waves in flows close to having a stagnation point at the bottom, for $p_{1}=-0.5$ and $\gamma_{1}=0:(a) \gamma_{2}=-3.23$ (below the gap); (c) $\gamma_{2}=-3.25$ (below the gap). The background current profiles corresponding to zero-amplitude waves are shown in $(b, d)$, respectively. In the plot of the particle paths, the grey lines indicate the final position of the particles if under zero-amplitude waves after the time $4 L / c$.

in the bottom layer. The initial positions are denoted by dots and the final positions are indicated by square markers.

First of all, we plot the computed particle trajectories in the moving frame, as illustrated in figure 18, which theoretically are the corresponding streamlines. The streamlines passing through the initial particle locations are plotted for comparison, and a good agreement is seen, suggesting the accuracy of the method for computing particle trajectories.

For an irrotational layer on a rotational layer, the particle trajectories in flows close to having a stagnation point at the crest are plotted in figure 19 and those in flows close to having a stagnation point at the bottom are plotted in figure 20 . We show that as theoretically predicted by Constantin (2006) when a particle reaches the crest of the wave in flows having a stagnation point at the crest, it does not pause there but moves further (figure 19a). However, for waves in flows close to having an internal stagnation point or a stagnation point at the bottom, a particle reaching the stagnation point tends to pause at that point in the moving frame, similar to the case shown in Ribeiro et al. (2017) for flows with a bottom stagnation point. Some observations on the features of the particle trajectories are listed below:

(i) In both figures 19 and 20, the horizontal positions of the particles are on the right of the grey lines (which are the positions of the particles with no waves), indicating that the waves induce a positive drift of the particles in the direction of the propagating waves. This is consistent with what was proved by Constantin (2012b) and the experimental results (Paprota \& Sulisz 2018) for irrotational flows.

(ii) In the top irrotational layer, the horizontal displacement (with respect to the background current induced displacement) or drift is larger when the particle is 

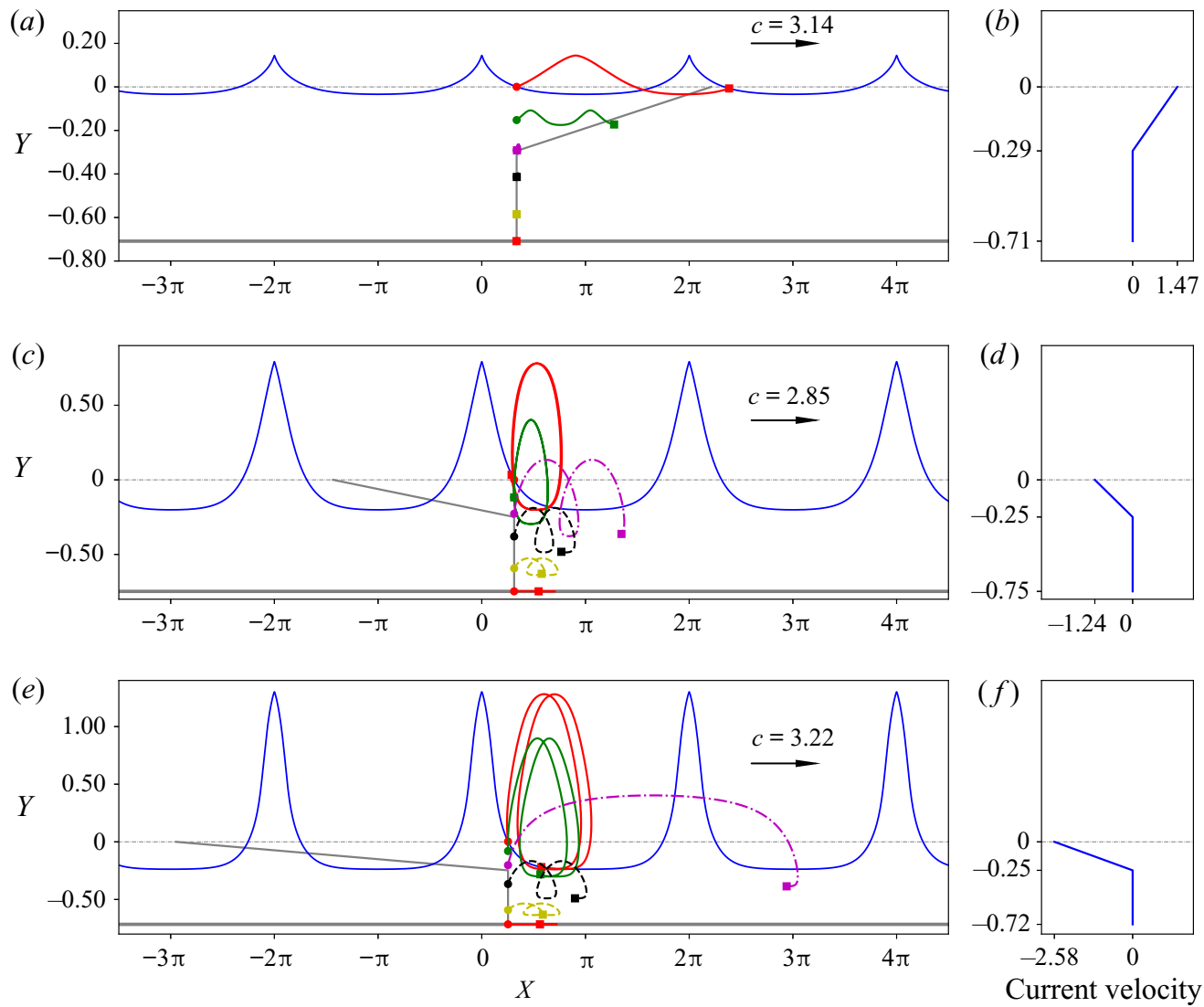

Figure 21. Particle trajectories under the waves in flows close to having a stagnation point near the crest, for $p_{1}=-0.7$ and $\gamma_{2}=0$ : (a) $\gamma_{1}=5 ;(c) \gamma_{1}=-5 ;$ (e) $\gamma_{1}=-10.42$. The background current profiles corresponding to zero-amplitude waves are shown in $(b, d, f)$, respectively. In the plot of the particle paths, the grey lines indicate the final position of the particles if under zero-amplitude waves after the time $4 L / c$.

closer to the surface than when it is near the interface of the two layers, which is consistent with experimental and approximate theoretical results (Curtis et al. 2018; Paprota \& Sulisz 2018). In the bottom rotational layer, when the vorticity is positive, the wave induced drift is larger when the particle is close to the interface as compared to the drift induced when the particle is close to the bottom surface. For negative (adverse) vorticity in the bottom layer, the largest drift tends to occur at the bottom surface (i.e. the bottom flat bed) when the flow is close to having a stagnation point at the bottom, as also observed in Ribeiro et al. (2017) for linear shear flow.

(iii) For flows close to having a stagnation point at the crest, as shown in figure 19, the negative vorticity in the bottom layer clearly increases the drift of the particles in the top irrotational layer relative to the background current, especially for particles at the surface.

(iv) For flows close to having a stagnation point at the bottom, as in figure 20, a stronger negative vorticity in the bottom layer tends to decrease the relative drifts of the particles at different vertical positions in the top layer, as observed by comparing figures 19(c) and 20(c). 

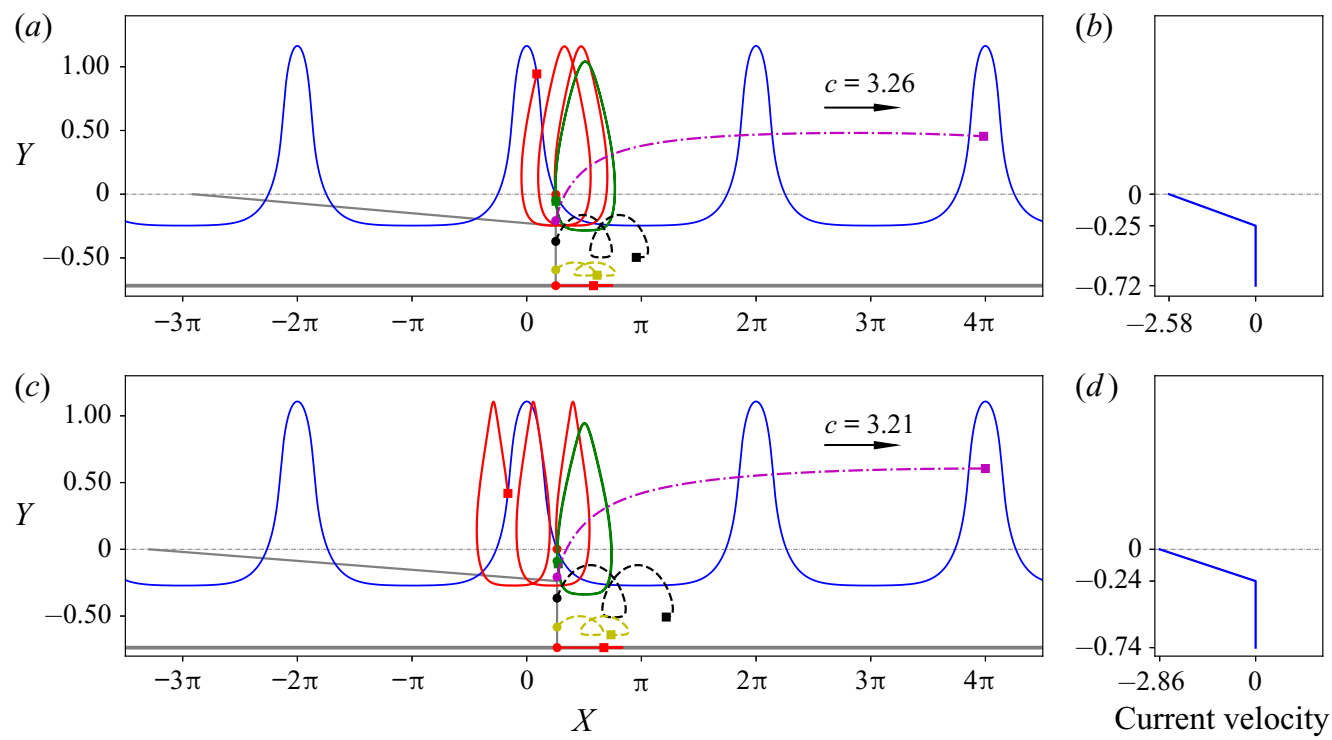

Figure 22. Particle trajectories under the waves in flows close to having a stagnation point at the bottom, for $p_{1}=-0.7$ and $\gamma_{2}=0$ : (a) $\gamma_{2}=-10.42$ (below the gap); (c) $\gamma_{2}=-12$. The background current profiles corresponding to zero-amplitude waves are shown in $(b, d)$, respectively. In the plot of the particle paths, the grey lines indicate the final position of the particles if under zero-amplitude waves after the time $4 L / c$.

With a shear layer on an irrotational layer, figure 21 shows the particle trajectories for flows close to having a stagnation point at the crest and figure 22 reveals the particle trajectories for flows close to having a stagnation point at the location when the vorticity jump occurs. Again, we can see from both figures that the wave-induced drifts are positive in the direction of the propagating waves. Some conspicuous features are observed as described below:

(i) In the bottom irrotational layer, the drift is always larger when the particle is close to the interface, consistent with particle paths in irrotational flow (Paprota \& Sulisz 2018).

(ii) In the top rotational layer, when the vorticity is positive, the wave-induced drift is larger when the particle is near the surface as compared to the drift when the particle is close to the interface of the two layers; while with negative vorticity, for flows close to having a stagnation point at the interface, the particle with the largest drift is at the interface.

(iii) A negative vorticity in the top layer tends to increase the wave-induced drifts of particles in both layers.

\section{Conclusions}

In this paper, we investigate the behaviour of flow along a new bifurcation curve, and the pressure and particle trajectories under waves travelling on a rotational flow with discontinuous vorticity (close to having a stagnation point). For this purpose, we have developed a method for computing the particle paths using the velocity field obtained from a numerical continuation method based on DJ transform of the Euler equations for periodic waves on rotational flow. The continuation method generates a group of waves of fixed relative mass flux until a point where a flow with a stagnation point is reached. 
By using the previously mentioned methods, we have recovered new branches of the bifurcation curves not connected to the trivial (laminar) solution, for waves on rotational flow with discontinuous vorticity. Particularly, we show that along the bifurcation curves, the stagnation point can first occur at the position where the vorticity jumps and then again occur at the surface. On the pressure distribution, we show that for the case of shear layer on an irrotational layer, the pressure in the top layer under the crest could be less than the atmosphere pressure and the pressure in the top layer near the interface displays a 'plateau' behaviour when the vorticity is large with a negative sign (i.e. adverse). This 'plateau' behaviour of the pressure has only been observed along the bottom for linear-shear flow. We further show the particle trajectories in flows close to having a stagnation point for various cases and qualitatively discuss the influence of vorticity of the rotational layer on the drifts of the particles in both layers.

Acknowledgements. The authors are grateful to the anonymous reviewers for their comments and suggestions which helped us improve the paper. L.C. acknowledges the partial support of the National Natural Science Foundation of China (grant number: 51978506). C.-I.M. acknowledges the support of the Austrian Science Fund (FWF) through research grant P 30878-N32.

Declaration of interests. The authors report no conflict of interest.

Author ORCIDs.

다 Lin Chen https://orcid.org/0000-0002-3570-234X;

(ㄱ) Biswajit Basu https://orcid.org/0000-0001-7740-7616;

() Calin-I. Martin https://orcid.org/0000-0002-5800-9265.

\section{REFERENCES}

ABLowitz, M.J., FoKas, A.S. \& Musslimani, Z.H. 2006 On a new non-local formulation of water waves. J. Fluid Mech. 562, 313-343.

Ali, A. \& KALISCH, H. 2013 Reconstruction of the pressure in long-wave models with constant vorticity. Eur. J. Mech. B/Fluids 37, 187-194.

AmANN, D. \& KAlimeris, K. 2018 A numerical continuation approach for computing water waves of large wave height. Eur. J. Mech. B/Fluids 67, 314-328.

BASU, B. 2017 Estimation of wave heights from pressure data at the bed in the presence of uniform underlying currents. Nonlinear Anal. 156, 82-89.

BASU, B. 2018 Wave height estimates from pressure and velocity data at an intermediate depth in the presence of uniform currents. Phil. Trans. R. Soc. Lond. A 376 (2111), 20170087.

BASU, B. 2019 On some properties of velocity field for two-dimensional rotational steady water waves. Nonlinear Anal. 184, 17-34.

Borluk, H. \& Kalisch, H. 2012 Particle dynamics in the KdV approximation. Wave Motion 49 (8), 691-709.

Carter, J.D., Curtis, C.W. \& Kalisch, H. 2020 Particle trajectories in nonlinear Schrödinger models. Water Waves 2, 31-57.

Chappelear, J.E. 1961 Direct numerical calculation of wave properties. J. Geophys. Res. 66 (2), 501-508.

CHEN, L. \& BASU, B. $2018 a$ Fatigue load estimation of a spar-type floating offshore wind turbine considering wave-current interactions. Intl J. Fatigue 116, 421-428.

CHEN, L. \& BASU, B. $2018 b$ Wave-current interaction effects on structural responses of floating offshore wind turbines. Wind Energy 22, 327-339.

Clamond, D. \& DuTYKh, D. 2018 Accurate fast computation of steady two-dimensional surface gravity waves in arbitrary depth. J. Fluid Mech. 844, 491-518.

Constantin, A. 2006 The trajectories of particles in Stokes waves. Invent. Math. 166 (3), 523-535.

Constantin, A. 2011 Nonlinear Water Waves with Applications to Wave-Current Interactions and Tsunamis. CMBS-NSF Reg. Conf. Ser. Appl. Math, vol. 81. SIAM.

Constantin, A. 2012a Dispersion relations for periodic traveling water waves in flows with discontinuous vorticity. Commun. Pure Appl. Anal. 11 (4), 1397-1406.

Constantin, A. $2012 b$ Particle trajectories in extreme stokes waves. J. Appl. Maths 77 (3), 293-307.

Constantin, A. 2015 The flow beneath a periodic travelling surface water wave. J. Phys. A 48 (14), 143001. 


\section{Chen, B. Basu and C.-I. Martin}

Constantin, A., Ehrnström, M. \& WAhlÉn, E. 2007 Symmetry of steady periodic gravity water waves with vorticity. Duke Math. J. 140 (3), 591-603.

Constantin, A., Kalimeris, K. \& Scherzer, O. 2015 a Approximations of steady periodic water waves in flows with constant vorticity. Nonlinear Anal. RWA 25, 276-306.

Constantin, A., Kalimeris, K. \& Scherzer, O. 2015b A penalization method for calculating the flow beneath traveling water waves of large amplitude. SIAM J. Appl. Maths 75 (4), 1513-1535.

Constantin, A. \& Strauss, W.A. 2004 Exact steady periodic water waves with vorticity. Commun. Pure Appl. Maths 57 (4), 481-527.

Constantin, A. \& Strauss, W.A. 2007 Rotational steady water waves near stagnation. Phil. Trans. R. Soc. Lond. A 365 (1858), 2227-2239.

Constantin, A. \& Strauss, W.A. 2010 Pressure beneath a Stokes wave. Commun. Pure Appl. Maths 63 (4), 533-557.

Constantin, A. \& Strauss, W.A. 2011 Periodic traveling gravity water waves with discontinuous vorticity. Arch. Rat. Mech. Anal. 202 (1), 133-175.

Constantin, A. \& Villari, G. 2008 Particle trajectories in linear water waves. J. Math. Fluid Mech. 10 (1), 1-18.

Curtis, C.W., Carter, J.D. \& Kalisch, H. 2018 Particle paths in nonlinear Schrödinger models in the presence of linear shear currents. J. Fluid Mech. 855, 322-350.

DAlRyMple, R.A. 1973 Water wave models and wave forces with shear currents. Tech. Rep. No. 2. Coastal and Oceanographic Engineering Laboratory, University of Florida.

DAlRymple, R.A. 1974 a A finite amplitude wave on a linear shear current. J. Geophys. Res. 79 (30), 4498-4504.

DALRYMPle, R.A. $1974 b$ Water waves on a bilinear shear current. In Proceedings of the Fourteenth Coastal Engineering Conference (ed. M.P. O’Brien), pp. 626-641. ASCE.

DALRYMPLE, R.A. 1977 A numerical model for periodic finite amplitude waves on a rotational fluid. J. Comput. Phys. 24 (1), 29-42.

DAlRyMPle, R.A. \& COX, J.C. 1976 Symmetric finite-amplitude rotational water waves. J. Phys. Oceanogr. $6(6), 847-852$.

DONG, Z. \& KIRBY, J.T. 2012 Theoretical and numerical study of wave-current interaction in strongly-sheared flows. In Proceedings of the 33rd Coastal Engineering Conference (ed. P. Lynett \& J.M. Smith), p. waves.2. ASCE.

Dubreil-JACotin, M.-L. 1934 Sur la détermination rigoureuse des ondes permanentes périodiques d'ampleur finie. J. Math. Pure. Appl. 13, 217-291.

DYACHENKO, S.A. \& HUR, V.M. 2019a Stokes waves with constant vorticity: folds, gaps and fluid bubbles. J. Fluid Mech. 878, 502-521.

DyACHENKO, S.A. \& HuR, V.M. $2019 b$ Stokes waves with constant vorticity: I. Numerical computation. Stud. Appl. Maths 142, 162-189.

EHRNSTRÖM, M. 2008 On the streamlines and particle paths of gravitational water waves. Nonlinearity 21 (5), 1141-1154.

Ehrnström, M., Escher, J. \& WAhlÉn, E. 2011 Steady water waves with multiple critical layers. SIAM J. Math. Anal. 43 (3), 1436-1456.

FoKAS, A.S. \& NACHBIN, A. 2012 Water waves over a variable bottom: a non-local formulation and conformal mappings. J. Fluid Mech. 695, 288-309.

FRANCIUS, M. \& KHARIF, C. 2017 Two-dimensional stability of finite-amplitude gravity waves on water of finite depth with constant vorticity. J. Fluid Mech. 830, 631-659.

Grue, J. \& KolaAs, J. 2017 Experimental particle paths and drift velocity in steep waves at finite water depth. J. Fluid Mech. 810, R1.

Grue, J., KolaAs, J. \& Jensen, A. 2014 Velocity fields in breaking-limited waves on finite depth. Eur. J. Mech. B/Fluids 47, 97-107.

GuenneBAuD, G. \& JACOB, B. 2010 Eigen v3. http://eigen.tuxfamily.org.

HENRY, D. 2006 The trajectories of particles in deep-water stokes waves. Intl Math. Res. Notices 2006, 23405.

Henry, D. $2013 a$ Large amplitude steady periodic waves for fixed-depth rotational flows. Commun. Part. Diff. Equ. 38 (6), 1015-1037.

Henry, D. $2013 b$ Steady periodic waves bifurcating for fixed-depth rotational flows. Q. Appl. Maths 71 (3), 455-487.

HuR, V.M. 2006 Global bifurcation theory of deep-water waves with vorticity. SIAM J. Math. Anal. 37 (5), 1482-1521.

Hur, V.M. 2011 Stokeswaves with vorticity. J. Anal. Maths 113 (1), 331-386.

KALIMERIS, K. 2018 Analytical approximation and numerical simulations for periodic travelling water waves. Phil. Trans. R. Soc. Lond. A 376 (2111), 20170093. 


\section{Rotational flows beneath periodic waves near stagnation}

KARAGEORGIS, P. 2012 Dispersion relation for water waves with non-constant vorticity. Eur. J. Mech. B/Fluids $34,7-12$.

KIRBY, J.T. 1984 Note on linear surface wave-current interaction over slowly varying topography. J. Geophys. Res. 89 (C1), 945-947.

KIRBY, J.T. \& CHEN, T.-M. 1989 Surface waves on vertically sheared flows: approximate dispersion relations. J. Geophys. Res. 94 (C1), 1013-1027.

KIRBY, J.T. \& DALRYMPlE, R.A. 1983 Propagation of weakly nonlinear surface waves in the presence of varying depth and currents. In Proceedings of the 20 IAHR Congress, pp. 198-202. IAHR.

Ko, J. \& StRAuss, W.A. 2008a Effect of vorticity on steady water waves. J. Fluid Mech. 608, $197-215$.

Ko, J. \& Strauss, W.A. 2008 b Large-amplitude steady rotational water waves. Eur. J. Mech. B/Fluids 27 (2), 96-109.

Longuet-Higgins, M.S. \& Stewart, R.W. 1961 The changes in amplitude of short gravity waves on steady non-uniform currents. J. Fluid Mech. 10 (4), 529-549.

MARTIN, C.I. 2015 Dispersion relations for gravity water flows with two rotational layers. Eur. J. Mech. B/Fluids 50, 9-18.

Matioc, A.-V. 2010 On particle trajectories in linear water waves. Nonlinear Anal. 11 (5), 4275-4284.

NACHBIN, A. \& RiBEIRO-JUNIOR, R. 2014 A boundary integral formulation for particle trajectories in stokes waves. Discrete Contin. Dyn. Syst. 34, 3135-3153.

Nachbin, A. \& Ribeiro-Junior, R. 2017 Capturing the flow beneath water waves. Phil. Trans. R. Soc. Lond. A 376 (2111), 20170098.

Oliveras, K.L., Vasan, V., Deconinck, B. \& Henderson, D. 2012 Recovering the water-wave profile from pressure measurements. SIAM J. Appl. Math. 72 (3), 897-918.

PAprota, M. \& Sulisz, W. 2018 Particle trajectories and mass transport under mechanically generated nonlinear water waves. Phys. Fluids 30 (10), 102101.

Paprota, M., Sulisz, W. \& RedA, A. 2016 Experimental study of wave-induced mass transport. J. Hydraul. Res. 54 (4), 423-434.

Ribeiro, R., Milewski, P.A. \& NACHBin, A. 2017 Flow structure beneath rotational water waves with stagnation points. J. Fluid Mech. 812, 792-814.

SEYDEL, R. 2009 Practical Bifurcation and Stability Analysis. Interdisciplinary Applied Mathematics, vol. 5. Springer Science and Business Media.

Simmen, J.A. \& Saffman, P.G. 1985 Steady deep-water waves on a linear shear current. Stud. Appl. Maths 73 (1), 35-57.

Strauss, W.A. 2010 Steady water waves. Bull. Am. Math. Soc. 47 (4), 671-694.

Strauss, W.A. 2012 Vorticity jumps in steady water waves. Discrete Cont. Dyn. Syst. B 17 (4), 1101-1112.

Swan, C., Cummins, I.P. \& JAMES, R.L. 2001 An experimental study of two-dimensional surface water waves propagating on depth-varying currents. Part 1. Regular waves. J. Fluid Mech. 428, 273-304.

Teles dA Silva, A.F. \& PEREgrine, D.H. 1988 Steep, steady surface waves on water of finite depth with constant vorticity. J. Fluid Mech. 195, 281-302.

ThomAs, G.P. 1990 Wave-current interactions: an experimental and numerical study. Part 2. Nonlinear waves. J. Fluid Mech. 216, 505-536.

UMEYAMA, M. 2012 Eulerian-Langrangian analysis for particle velocities and tracjectories in a pure wave motion using particle image velocimetry. Phil. Trans. R. Soc. Lond. A 370 (1858), 1687-1702.

VAn Den Bremer, T.S. \& BreiviK, Ø. 2017 Stokes drift. Phil. Trans. R. Soc. Lond. A 376 (2111), 20170104.

VAN DEN BREMER, T.S. \& TAYLOR, P.H. 2016 Lagrangian transport for two-dimensional deep-water surface gravity wave groups. Proc. R. Soc. Lond. A 472 (2192), 20160159.

VARVARUCA, E. 2008 On some properties of traveling water waves with vorticity. SIAM J. Math. Anal. 39 (5), 1686-1692.

VASAn, V. \& Oliveras, K. 2014 Pressure beneath a traveling wave with constant vorticity. Discrete Cont. Dyn. Syst. A 34 (8), 3219-3239.

WAHLÉn, E. 2009 Steady water waves with a critical layer. J. Differ. Equ. 246 (6), 2468-2483. 